\title{
Bipolar Ionization Cones in the Extended Narrow-line Region of Nearby QSO2s
}

\author{
T. Storchi-Bergmann ${ }^{1,2}$ (1) , B. Dall'Agnol de Oliveira ${ }^{1}$, L. F. Longo Micchi ${ }^{3}$, H. R. Schmitt ${ }^{4}$ (1) , T. C. Fischer ${ }^{5}$ (1) S. Kraemer ${ }^{3}$ (1), \\ M. Crenshaw ${ }^{6}$ (D) P. Maksym ${ }^{2}$ (D), M. Elvis ${ }^{2}$ (D), G. Fabbiano ${ }^{2}$ (D), and L. Colina ${ }^{7}$ \\ ${ }^{1}$ Departamento de Astronomia, Universidade Federal do Rio Grande do Sul, IF, CP 15051, 91501-970 Porto Alegre, RS, Brazil; thaisa@ufrgs.br \\ ${ }^{2}$ Harvard-Smithsonian Center for Astrophysics, 60 Garden St., Cambridge, MA 02138, USA \\ ${ }^{3}$ Institute for Astrophysics and Computational Sciences, Department of Physics, The Catholic University of America, Washington, DC 20064, USA \\ ${ }^{4}$ Naval Research Laboratory, Washington, DC 20375, USA \\ ${ }^{5}$ Goddard Space Flight Center, 8800 Greenbelt Rd., Greenbelt, MD 20771, USA \\ ${ }^{6}$ Department of Physics and Astronomy, Georgia State University, Astronomy Offices, 25 Park Place, Suite 600, Atlanta, GA 30303, USA \\ ${ }^{7}$ Centro de Astrobiologa (CAB, CSIC-INTA), Carretera de Ajalvir, E-28850 Torrejn de Ardoz, Madrid, Spain \\ Received 2018 May 16; revised 2018 October 1; accepted 2018 October 9; published 2018 November 14
}

\begin{abstract}
We have used narrowband [O III] $\lambda \lambda 4959,5007$ and $\mathrm{H} \alpha+[\mathrm{N} \mathrm{II}] \lambda \lambda 6548,84$ Hubble Space Telescope (HST) images of nine luminous $\left(L[\mathrm{O} \mathrm{III}]>10^{42} \mathrm{erg} \mathrm{s}^{-1}\right.$ ) type 2 QSOs with redshifts $0.1<z<0.5$ in order to constrain the geometry of their extended narrow-line regions (ENLRs), as recent ground-based studies suggest that these regions become more spherical at high luminosities due to destruction of the torus. We instead find elongated ENLRs reaching 4-19 kpc from the nucleus and bipolar ionization cones in $[\mathrm{O} \mathrm{III]} /(\mathrm{H} \alpha+[\mathrm{N} \mathrm{II}])$ excitation maps indicating that the torus survives these luminosities, allowing the escape of $\approx 10$ times higher ionizing photon rates along the ionization axis than perpendicular to it. The exceptional HST angular resolution was key to our success in arriving at these conclusions. Combining our measurements with previous ones based on similar $H S T$ data, we have revisited the relation between the ENLR radius $R_{\text {maj }}$ and $L[\mathrm{O}$ III $]$ over the range $39<\log \left(L[\mathrm{O}\right.$ III] $)<43.5\left(L\right.$ in $\left.\operatorname{erg~s}^{-1}\right): \log \left(R_{\text {maj }}\right)=(0.51 \pm 0.03) \log (L[\mathrm{O}$ III] $)$ $-18.12 \pm 0.98$. The radius of the ENLR keeps increasing with $L[\mathrm{O}$ III] in our data, implying that the ENLR can extend to distances beyond the limit of the galaxy if gas is present there-e.g., from active galactic nucleus (AGN) outflows or interactions, seen in six objects of our sample. We attribute the flattening previously seen in this relation to the fact that the ENLR is matter-bounded, meaning that ionizing photons usually escape to the intergalactic medium in luminous AGNs. Estimated ionized gas masses of the ENLRs range from 0.3 to $2 \times 10^{8} M_{\odot}$, and estimated powers for associated outflows range from $<0.1 \%$ to a few percent of the QSO luminosity.
\end{abstract}

Key words: galaxies: active - galaxies: jets - galaxies: Seyfert - ISM: jets and outflows - quasars: emission lines quasars: supermassive black holes

\section{Introduction}

The physical processes that couple the growth of supermassive black holes (SMBHs) to their host galaxies occur in the vicinity of the galaxy nucleus $(\approx$ inner kpc; Hopkins \& Quataert 2010) when it becomes active due to mass accretion onto the SMBH (Ferrarese \& Ford 2005; Kormendy \& Ho 2013). The radiation emitted by active galactic nuclei (AGNs) works as a flashlight that illuminates and ionizes the gas in the vicinity of the nucleus, forming the narrow-line region (NLR). The AGN-driven winds (Elvis 2000; Ciotti et al. 2010; StorchiBergmann et al. 2010; Barbosa et al. 2014) interact with the gas and produce outflows reaching velocities of hundreds of $\mathrm{km} \mathrm{s}^{-1}$ (Storchi-Bergmann et al. 2010; Fischer et al. 2013). Relativistic jets emanating from the AGN also interact with the gas of the NLR (Wang et al. 2009; Couto et al. 2017). Both types of outflow produce feedback, which has been an important ingredient in galaxy evolution models to avoid producing overmassive galaxies (Fabian 2012). Inflows have also been observed in this region (Riffel et al. 2008, 2013; Crenshaw et al. 2010; Schnorr-Müller et al. 2014b). The importance of the NLR stems from the fact that it is spatially resolved, as it extends from hundreds to thousands of pc from the nucleus and exhibits strong line emission. These properties of the NLR allow the observation of the interaction between the AGN and the circumnuclear gas in the galaxy (1) via the observation of the NLR geometry and excitation properties that constrain the AGN structure and nature of the ionizing source and (2) via the gas kinematics that maps the processes of the AGN feeding and feedback.

Over the last $30 \mathrm{yr}$, narrowband imaging of the NLR became an important tool in the study of the ionization mechanism, morphology, and implied geometry of the AGN. Starting with ground-based observations of Seyfert (Sy) galaxies (e.g., Haniff et al. 1988; Pogge 1988; Wilson \& Tsvetanov 1994), emission-line images of [O III] $\lambda 5007$ and $\mathrm{H} \alpha+[\mathrm{N}$ II] showed that the NLRs of several Sy galaxies have biconical shapes, with the apex at the nucleus. These observations were of key importance in validating the unified model of AGNs (Antonucci 1993), in which both Sy types have an SMBH fed by an accretion disk surrounded by a torus of molecular gas and dust that collimates the ionizing radiation, leading to the observed biconical shape. However, although these groundbased narrowband images of Sy galaxies have allowed the detection of shadowing of the nuclear ionizing radiation, they could resolve only the nearest and brightest sources. For fainter and more distant sources, the NLR angular diameters decrease below the resolution limit of ground-based telescopes $\left(\sim 1^{\prime \prime}\right)$. The use of the Hubble Space Telescope (HST) to image the NLR was crucial for the further development of the field (e.g., Wilson et al. 1993; Capetti et al. 1996; Falcke et al. 1998; Ferruit et al. 2000; Schmitt et al. 2003b). The high spatial resolution of these observations allowed better constraints on the unified model and the study of the NLR structure in detail. Some results from these studies include the alignment of the radio jet and NLR, which indicates that the torus and accretion 
disk are connected, the radio jet can significantly influence the NLR emission, and Sy 1 NLRs are statistically more circular and concentrated than Sy 2s, which can be attributed to foreshortening in the former (Schmitt et al. 2003a).

Another important result from these studies-in particular, of the snapshot survey of Schmitt et al. (2003a, 2003b) —was the detection of a relation between the NLR radius $R_{\mathrm{NLR}}$ and its luminosity $L([\mathrm{O} \mathrm{III}])$. For a sample of 60 AGNs with $L([\mathrm{O} \mathrm{III}])<10^{42} \mathrm{erg} \mathrm{s}^{-1}$, these authors found the relation $R_{\mathrm{NLR}} \propto L[\mathrm{O} \mathrm{III}]^{0.33}$. This was interpreted as due to the fact that the ionization parameter is not constant along the NLR and that most of the [O III] emission comes from a low-density region. A constant ionization parameter had been suggested by previous observations of the NLR of a sample of QSOs that implied a slope of $\sim 0.5$ for the relation above (Bennert et al. 2002) but that was later concluded to lead to predicted NLR sizes much larger than observed (Netzer et al. 2004).

Recent results obtained via integral field spectroscopy (e.g., Liu et al. 2013) have suggested that at higher redshifts and luminosities, the geometry of the AGN outflows may change, becoming less collimated and more spherical, due to the higher AGN luminosity and higher power of the possibly associated outflow that would clear a larger area of the dusty toroidal region surrounding the accretion disk and broad-line region (BLR; Elitzur et al. 2014). But, as these studies have been done using ground-based telescopes, for $z \geqslant 0.1$, the limited angular resolution and smearing by the seeing precludes firm constraints on the NLR (or ENLR) morphology. In order to provide such constraints at higher redshifts and AGN luminosities, we have obtained narrowband [O III] $\lambda 5007$ and $\mathrm{H} \alpha+[\mathrm{N} \mathrm{II}]$ images with the HST of a flux-limited sample of nine luminous type 2 AGNs (or QSOs, due to their high luminosities) with redshifts in the range $0.1 \leqslant z \leqslant 0.5$. We use these images to obtain excitation maps, through the ratio $[\mathrm{O} \mathrm{III]}] /(\mathrm{H} \alpha+[\mathrm{N} \mathrm{II}])$, ionized gas masses from the $\mathrm{H} \alpha$ luminosities and extend the relation between the ENLR radius $R_{\text {maj }}$ and $L[\mathrm{O}$ III $]$ to the range $39<\log (L[\mathrm{O} \mathrm{III}])<43.5$ ( $L$ in $\left.\operatorname{erg~s}^{-1}\right)$.

This paper is organized as follows. In Section 2, we present the sample; in Section 3, we discuss the observations, data reduction and measurements; and in Section 4, we present the images and ionization maps, as well as total gas masses and spatial profiles of the ionized gas mass surface density. In Section 5, we discuss our results, and in Section 6, we present our conclusions.

\section{Sample}

Our sample was selected from the catalog of QSO2 galaxies of Reyes et al. (2008), which comprises 887 galaxies with $0.04<z<0.8$ and is thus just beyond the redshift range of the HST imaging survey of Schmitt et al. (2003b). The selection was done according to the following criteria. (1) The galaxies should have [O III] fluxes larger than $1.5 \times 10^{-14} \mathrm{erg} \mathrm{cm}^{-2} \mathrm{~s}^{-1}$ in order to assure enough signal-to-noise ratio in the narrowband images. (2) They should have redshifts in the range $0.1<z \leqslant 0.5$, thus allowing us to probe more luminous AGNs than in the previous study of Schmitt et al. (2003b). The luminous sources at $z \approx 0.4$ are predicted to have NLR sizes of $\sim 3 \mathrm{kpc}$ that correspond to angular sizes of about $0 . " 6$ and are thus resolvable down to scales of hundreds of pc at the host galaxy with HST. (3) They should have L[O III] values larger than $\log \left[L[\mathrm{O}\right.$ III $]=42.5\left(\right.$ in $\left.\operatorname{erg~s}^{-1}\right)$.
Table 1

Sample Properties

\begin{tabular}{lcccc}
\hline \hline ID & Name & $z$ & Scale & $D_{L}$ \\
$(1)$ & $(2)$ & $(3)$ & $(4)$ & $(5)$ \\
\hline 1 & $\mathrm{~J} 082313.50+313203.7$ & 0.433 & $5.46 \pm 0.25$ & $2310 \pm 110$ \\
2 & $\mathrm{~J} 084135.04+010156.3$ & 0.111 & $1.95 \pm 0.09$ & $498 \pm 24$ \\
3 & $\mathrm{~J} 085829.58+441734.7$ & 0.454 & $5.61 \pm 0.25$ & $2450 \pm 120$ \\
4 & $\mathrm{~J} 094521.34+173753.3$ & 0.128 & $2.22 \pm 0.11$ & $583 \pm 26$ \\
5 & $\mathrm{~J} 110952.82+423315.6$ & 0.262 & $3.91 \pm 0.18$ & $1284 \pm 58$ \\
6 & $\mathrm{~J} 113710.77+573158.7$ & 0.395 & $5.16 \pm 0.24$ & $2076 \pm 95$ \\
7 & $\mathrm{~J} 123006.79+394319.3$ & 0.407 & $5.26 \pm 0.23$ & $2149 \pm 93$ \\
8 & $\mathrm{~J} 135251.21+654113.2$ & 0.206 & $3.26 \pm 0.16$ & $980 \pm 47$ \\
9 & $\mathrm{~J} 155019.95+243238.7$ & 0.143 & $2.42 \pm 0.12$ & $653 \pm 32$ \\
\hline
\end{tabular}

Note. (1) Galaxy identification number used throughout the paper; (2) galaxy identification (without SDSS prefix); (3) redshift; (4) scale (in kpc $\operatorname{arcsec}^{-1}$ ); (5) luminosity distance (in Mpc).

The list of sample galaxies, together with their redshifts, angular scales (obtained from the angular distances), and luminosity distances $\left(D_{L}\right)$, are shown in Table 1 . The listed redshifts and the other derived quantities were obtained from the NASA/IPAC Extragalactic Database (NED). The angular and luminosity distances were corrected to the cosmic microwave background radiation reference frame. The uncertainties shown in the quantities listed in Table 1 correspond to the maximum errors resulting from the uncertainties in $H_{0}$ and $z$. The spatial scales range from 1.95 to $5.46 \mathrm{kpc} \operatorname{arcsec}^{-1}$ allowing a spatial resolution at the galaxies of $95-280 \mathrm{pc}$ with $H S T$.

We show in Figure 1 the SDSS spectra of the sample galaxies, together with curves showing the narrow filter passbands (see next section) used to obtain the line emission images centered on [O III] $\lambda 5007$ and $\mathrm{H} \alpha+[\mathrm{N} \mathrm{II}]$, as well as the broadband filter passbands used to obtain images in the continuum. For a few galaxies, the SDSS spectra did not cover the $\mathrm{H} \alpha+[\mathrm{N} \mathrm{II}]$ emission lines, as their redshifts put these lines beyond the upper wavelength limit of the SDSS spectra. The passbands were obtained with the Python package Astrolib PySynphot, which produces a synthetic throughput of the filter.

\section{Observations and Data Reduction}

The sample galaxies were observed with the HST Advanced Camera for Surveys (ACS) using linear ramp filters centered on the redshifted [O III] $\lambda 5007$ and $\mathrm{H} \alpha+[\mathrm{N}$ II] $\lambda 6548,84$ emission lines, as well as a medium or wideband filter centered in the continuum between these two sets of lines used to subtract the continuum contribution underneath the emission lines. The log of the observations, executed under program GO-13741 (PI: Storchi-Bergmann), is shown in Table 2.

The filter passbands are illustrated in Figure 1, together with the SDSS spectra of each galaxy. It can be seen that, for all sources, the [O III] filter well covers the $5007 \AA$ emission line but only partially covers the $4959 \AA$ line.

Data reduction was performed using tasks in the Image Reduction and Analysis Facility (IRAF). First, the set of images in each line were aligned with imalign and combined using crrej, which allows cosmic-ray removal, and subsequently divided by the exposure time (header keyword EXPTIME). Then, the image was multiplied by the header keyword PHOTFLAM to transform its units to erg cm $\mathrm{cm}^{-2} \mathrm{~s}^{-1} \AA^{-1}$. In order to allow for a precise continuum subtraction from the emission-line images, the line 

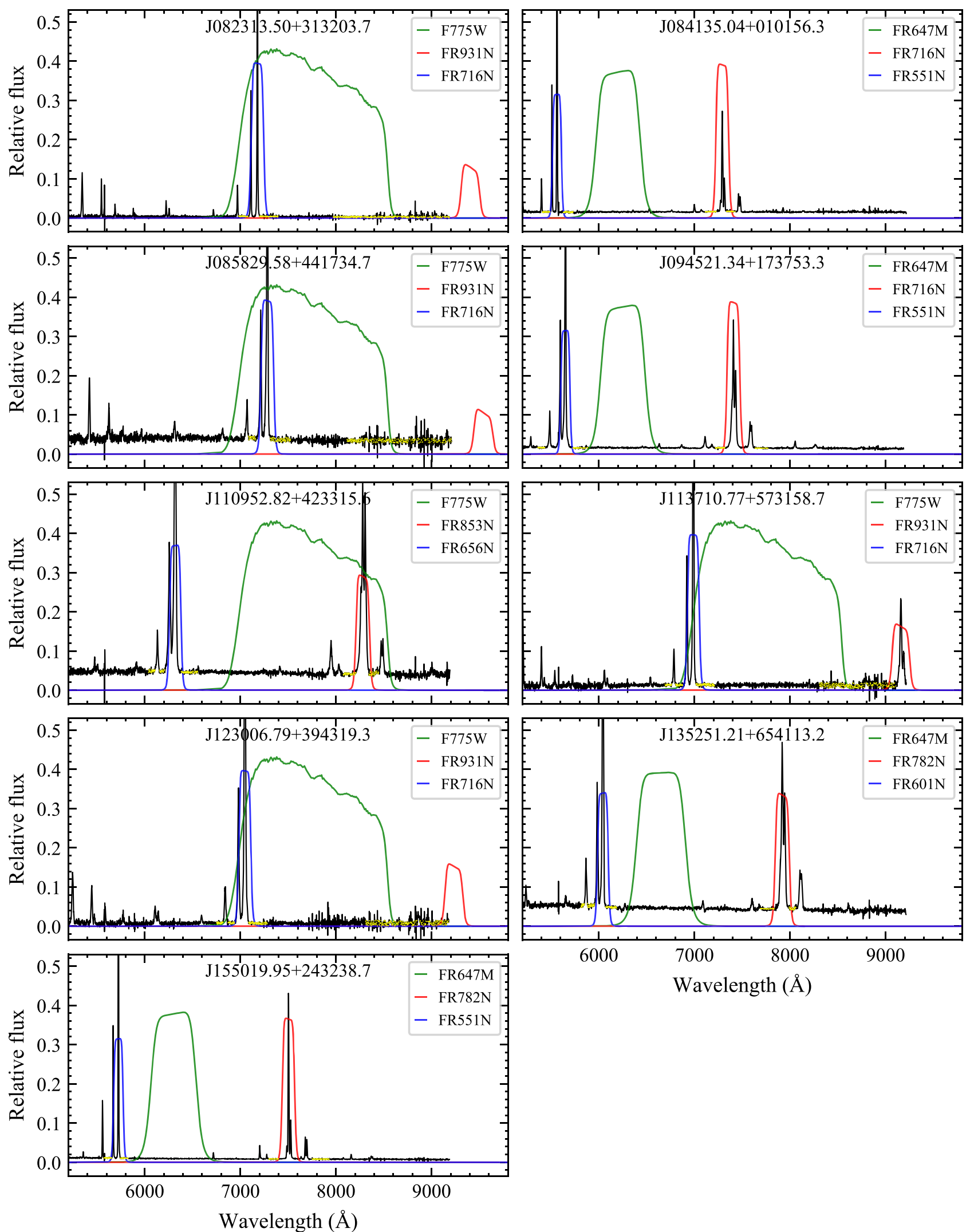

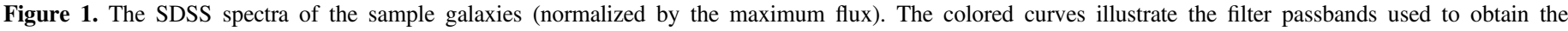

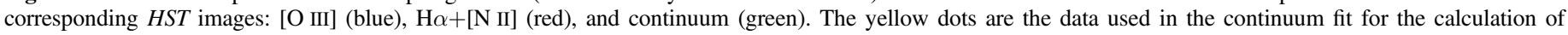
$S_{\mathrm{C},[\mathrm{O} \text { III }]}$ and $S_{\mathrm{C},[\mathrm{N} \mathrm{II}]+\mathrm{H} \alpha}$. 
Table 2

Observation Log

\begin{tabular}{|c|c|c|c|c|c|}
\hline $\begin{array}{l}\text { ID } \\
(1)\end{array}$ & $\begin{array}{l}\text { Name } \\
(2)\end{array}$ & $\begin{array}{c}\text { Date } \\
(3)\end{array}$ & $\begin{array}{l}\text { Filter } \\
(4)\end{array}$ & $\begin{array}{l}\text { Exptime } \\
\text { (5) }\end{array}$ & $\begin{array}{c}\text { Region } \\
\text { (6) }\end{array}$ \\
\hline 1 & J082313 & $\begin{array}{c}2015 \text { Jan } 01 \\
2014 \text { Dec } 31 \\
2015 \text { Jan } 01\end{array}$ & $\begin{array}{l}\text { F775W } \\
\text { FR931N } \\
\text { FR716N }\end{array}$ & $\begin{array}{c}200 \\
2542 \\
1866\end{array}$ & $\begin{array}{c}\text { Cont. } \\
\mathrm{H} \alpha+[\mathrm{N} \mathrm{II}] \\
{[\mathrm{O} \text { III }]}\end{array}$ \\
\hline 2 & J084135 & $\begin{array}{l}2015 \text { Feb } 16 \\
2015 \text { Feb } 16 \\
2015 \text { Feb } 16\end{array}$ & $\begin{array}{l}\text { FR647M } \\
\text { FR716N } \\
\text { FR551N }\end{array}$ & $\begin{array}{c}200 \\
2506 \\
2020\end{array}$ & $\begin{array}{c}\text { Cont. } \\
\mathrm{H} \alpha+[\mathrm{N} \mathrm{II}] \\
{[\mathrm{O} \text { III }]}\end{array}$ \\
\hline 3 & J085829 & $\begin{array}{l}2015 \text { Feb } 08 \\
2015 \text { Feb } 08 \\
2015 \text { Feb } 08\end{array}$ & $\begin{array}{l}\text { F775W } \\
\text { FR931N } \\
\text { FR716N }\end{array}$ & $\begin{array}{c}200 \\
2600 \\
1927\end{array}$ & $\begin{array}{c}\text { Cont. } \\
\mathrm{H} \alpha+[\mathrm{N} \mathrm{II}] \\
{[\mathrm{O} \text { III }]}\end{array}$ \\
\hline 4 & J094521 & $\begin{array}{l}2015 \text { Apr } 22 \\
2015 \text { Apr } 22 \\
2015 \text { Apr } 22\end{array}$ & $\begin{array}{l}\text { FR647M } \\
\text { FR716N } \\
\text { FR551N }\end{array}$ & $\begin{array}{c}200 \\
2516 \\
2031\end{array}$ & $\begin{array}{c}\text { Cont. } \\
\mathrm{H} \alpha+[\mathrm{N} \mathrm{II}] \\
{[\mathrm{O} \text { III }]}\end{array}$ \\
\hline 5 & J110952 & $\begin{array}{l}2015 \text { Feb } 16 \\
2015 \text { Feb } 16 \\
2015 \text { Feb } 16\end{array}$ & $\begin{array}{l}\text { F775W } \\
\text { FR853N } \\
\text { FR656N }\end{array}$ & $\begin{array}{c}200 \\
2600 \\
2029\end{array}$ & $\begin{array}{c}\text { Cont. } \\
\mathrm{H} \alpha+[\mathrm{N} \mathrm{II}] \\
{[\mathrm{O} \text { III }]}\end{array}$ \\
\hline 6 & $\mathrm{~J} 113710$ & $\begin{array}{l}2015 \text { Sep } 08 \\
2015 \text { Sep } 08 \\
2015 \text { Sep } 08\end{array}$ & $\begin{array}{l}\text { F775W } \\
\text { FR931N } \\
\text { FR716N }\end{array}$ & $\begin{array}{c}200 \\
2754 \\
2081\end{array}$ & $\begin{array}{c}\text { Cont. } \\
\mathrm{H} \alpha+[\mathrm{N} \mathrm{II}] \\
{[\mathrm{O} \text { III }]}\end{array}$ \\
\hline 7 & $\mathrm{~J} 123006$ & $\begin{array}{l}2015 \text { Jul } 04 \\
2015 \text { Jul } 04 \\
2015 \text { Jul } 04\end{array}$ & $\begin{array}{l}\text { F775W } \\
\text { FR931N } \\
\text { FR716N }\end{array}$ & $\begin{array}{c}200 \\
2562 \\
1889\end{array}$ & $\begin{array}{c}\text { Cont. } \\
\mathrm{H} \alpha+[\mathrm{N} \mathrm{II}] \\
{[\mathrm{O} \text { III }]}\end{array}$ \\
\hline 8 & $\mathrm{~J} 135251$ & $\begin{array}{l}2015 \text { May } 13 \\
2015 \text { May } 13 \\
2015 \text { May } 13\end{array}$ & $\begin{array}{l}\text { FR647M } \\
\text { FR782N } \\
\text { FR601N }\end{array}$ & $\begin{array}{c}200 \\
1786 \\
1976\end{array}$ & $\begin{array}{c}\text { Cont. } \\
\mathrm{H} \alpha+[\mathrm{N} \text { II }] \\
{[\mathrm{O} \text { III }]}\end{array}$ \\
\hline 9 & J155019 & $\begin{array}{l}2015 \text { Jun } 17 \\
2015 \text { Jun } 17 \\
2015 \text { Jun } 17\end{array}$ & $\begin{array}{l}\text { FR647M } \\
\text { FR782N } \\
\text { FR551N }\end{array}$ & $\begin{array}{c}200 \\
2516 \\
1875\end{array}$ & $\begin{array}{c}\text { Cont. } \\
\mathrm{H} \alpha+[\mathrm{N} \text { II }] \\
{[\mathrm{O} \text { III }]}\end{array}$ \\
\hline
\end{tabular}

Note. (1) ID used in the paper; (2) galaxy name; (3) observation date; (4) HST ACS filter; (5) exposure time (in s); (6) spectral region, where "Cont." means continuum.

images were aligned to the continuum images. In order to do this, the emission-line images were rotated using the rotate task, matching the continuum ORIENTAT header keyword, and subsequently aligned with the continuum image with the task imalign. After this, the sky contribution-obtained as the center of a Gaussian fit to the flux histogram distribution of a region devoid of galaxy contribution-was subtracted from the image. For each image pixel, we assume a flux uncertainty equal to three times the standard deviation of this Gaussian $\left(\sigma_{\text {sky }}\right)$.

As can be observed in Figure 1, in a number of cases, the wide-/median-band filter used to obtain the continuum images also includes some contribution of emission lines. In order to correct for this contamination, we have used the SDSS spectra to calibrate our continuum images by matching the continuum fluxes in the images to those of the SDSS spectrum (that corresponds to a circular aperture with diameter of $3^{\prime \prime}$ ). This was done as follows. We fitted the SDSS continuum under the emission line [O III] $\lambda 4959+\lambda 5007$, keeping only data differing by less than $2 \sigma$ of the average in the region. We then obtained the corresponding value from the HST continuum image $\left(C_{\text {circ,[O III }]}\right)$ by integrating the flux within a circular aperture with a diameter of $3^{\prime \prime}$ to match the SDSS aperture. The ratio $S_{\mathrm{C},[\mathrm{O} \text { III }]}=\mathrm{C}_{\mathrm{SDSS},[\mathrm{O} \text { III }]} / \mathrm{C}_{\text {circ, [O III }]}$ gives the value used to scale the continuum image before subtracting it from the [O III] $\lambda 4959+\lambda 5007$ image. The same procedure was performed to

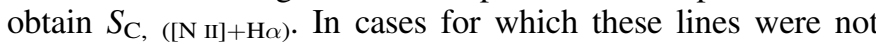
present in the spectra, we fitted a large region of the continuum in the red end of the spectrum (close to the missing lines).
These values are presented in Table 3, where the uncertainties in the SDSS continuum values were adopted as the standard deviation of the data used in the fit. These data are highlighted as yellow dots in Figure 1 .

Table 3 shows that the smallest-scale factors used to correct the continuum images for the emission-line contributionwhich correspond to the largest corrections due to emissionline contamination-were obtained for targets 7, 1, 6, and 3 (from the largest to the lowest corrections). In these cases, the continuum filter includes an important contribution from the [O III] emission lines, ranging from $60 \%$ to $20 \%$ of the total flux.

As mentioned above, the [O III] filter did not entirely cover the $\lambda 4959$ emission line. In order to obtain [O III] images including the total flux of both the $\lambda 4959$ and the $\lambda 5007$ emission lines, we proceeded as follows. First, we subtracted the fitted continuum from each SDSS spectrum. Then, we multiplied the result by the respective [O III] filter throughput and integrated in order to obtain the flux $F[\mathrm{O} \mathrm{III}]_{1}$ that would be obtained using the [O III] filter. Next, we modified the filter throughput, stretching the profile from the center by $50 \AA$ toward smaller wavelengths, in order to cover both emission lines. Now, the new integrated flux $\left(F[\mathrm{O} \mathrm{III}]_{2}\right)$ corresponds to what would be expected if the filter covered both emission lines. The ratio $r_{[\mathrm{O} I I I]}=F[\mathrm{O} \mathrm{III}]_{2} / F[\mathrm{O} \mathrm{III}]_{1}$ gives the multiplication factor used to make the narrowband [O III] images to include the sum of the $[\mathrm{O}$ III $] \lambda 5007+\lambda 4959$ emission-line fluxes.

In summary, the flux of each emission line ([O III] and [N II] $+\mathrm{H} \alpha$ ) was obtained as $F=F^{*}-S_{\mathrm{C}} F_{\mathrm{C} *}$, where $F^{*}$ is the flux before the continuum subtraction, $F$ is the flux after the subtraction, and $F_{C}$ is the continuum flux before the multiplication by the $S_{\mathrm{C}}$ scale. In the case of the [O III] image, the emission-line flux was also multiplied by $\left.r_{[\mathrm{O}} \mathrm{III}\right]$.

The resulting flux uncertainty per pixel in each line 1 is the quadrature sum of each contribution $\sigma_{1}^{2}=\sigma_{1^{*}}^{2}+\left(S_{\mathrm{C}, 1} \sigma_{\mathrm{C}^{*}}\right)^{2}+$ $\left(\sigma_{\mathrm{C}_{\mathrm{C}, 1}} F_{\mathrm{C}^{*}}\right)^{2}$, where $\sigma_{1^{*}}$ and $\sigma_{1}$ are the uncertainties before and after the continuum subtraction, $\sigma_{S_{\mathrm{C}, 1}}$ is the continuum scale uncertainty, and $\sigma_{\mathrm{C}^{*}}$ is the continuum uncertainty. Both $\sigma_{1^{*}}$ and $\sigma_{\mathrm{C}}^{*}$ are adopted as $3 \sigma_{\text {sky }}$ of the respective image. In the case of $l=[\mathrm{O} \mathrm{III}]$, the uncertainty in $\left.r_{[\mathrm{O}} \mathrm{III}\right]$ was also propagated. When a flux integration is performed, the errors of each pixel are added in quadrature.

In order to check the flux calibration after the above corrections, we integrated the resulting [O III] HST image flux over the SDSS circular aperture $\left(F[\mathrm{O} I I I]_{\text {circ }}\right)$ and compared it with $F[\mathrm{O} \mathrm{III}]_{\mathrm{SDSS}}$, the flux of both [O III] emission lines in the SDSS spectra. These values are displayed in Table 5, and a comparison between them is shown in Figure 2, along with a dashed line showing the loci of equal values. It can be seen that the fluxes agree with each other within the uncertainties, supporting the robustness of our reduction and calibration processes.

The bandwidths $\left(\Delta_{l}\right)$ of each filter were obtained from Astrolib PySynphot. They correspond to the width of a boxlike throughput curve, with the same area of the bandpass profile and height equal to the throughput at the average wavelength of the bandpass (avgwave in PySynphot). The continuum and the [O III] and $\mathrm{H} \alpha+[\mathrm{N} \mathrm{II}]$ narrowband images were multiplied by the corresponding bandwidth when fluxes in units of

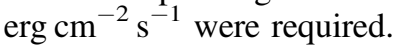


Table 3

Parameters Used in the Calibration of the Data

\begin{tabular}{|c|c|c|c|c|c|c|c|}
\hline $\begin{array}{l}\text { ID } \\
(1)\end{array}$ & $\begin{array}{l}\text { Name } \\
(2)\end{array}$ & $\begin{array}{l}\Delta_{C} \\
(3)\end{array}$ & $\begin{array}{c}\Delta_{[\mathrm{N} \mathrm{II]}+\mathrm{H} \alpha} \\
(4)\end{array}$ & $\begin{array}{l}\Delta_{[\mathrm{O} \text { III] }} \\
\quad(5)\end{array}$ & $\begin{array}{c}S_{\mathrm{C},[\mathrm{O} \text { III }]} \\
(6)\end{array}$ & $\begin{array}{c}\mathrm{S}_{\mathrm{C},[\mathrm{N} \mathrm{II]}+\mathrm{H} \alpha} \\
(7)\end{array}$ & $\begin{array}{c}r_{[\mathrm{O} \text { III] }} \\
(8)\end{array}$ \\
\hline 1 & J082313 & 1454.59 & 200.44 & 136.96 & $0.46 \pm 0.09$ & $0.3 \pm 0.1$ & $1.16 \pm 0.02$ \\
\hline 2 & J084135 & 482.21 & 139.54 & 98.78 & $0.98 \pm 0.05$ & $1.06 \pm 0.04$ & $1.20 \pm 0.02$ \\
\hline 3 & J085829 & 1454.59 & 204.58 & 139.33 & $0.79 \pm 0.07$ & $0.68 \pm 0.06$ & $1.17 \pm 0.02$ \\
\hline 4 & J094521 & 489.11 & 141.00 & 101.72 & $0.91 \pm 0.04$ & $0.89 \pm 0.04$ & $1.19 \pm 0.02$ \\
\hline 5 & J110952 & 1454.59 & 147.79 & 126.34 & $0.91 \pm 0.05$ & $0.81 \pm 0.05$ & $1.15 \pm 0.02$ \\
\hline 6 & $\mathrm{~J} 113710$ & 1454.59 & 191.44 & 131.02 & $0.71 \pm 0.09$ & $0.6 \pm 0.2$ & $1.17 \pm 0.02$ \\
\hline 7 & $\mathrm{~J} 123006$ & 1454.59 & 195.50 & 133.06 & $0.4 \pm 0.1$ & $0.5 \pm 0.2$ & $1.16 \pm 0.02$ \\
\hline 8 & $\mathrm{~J} 135251$ & 538.57 & 154.73 & 118.60 & $0.95 \pm 0.06$ & $0.84 \pm 0.03$ & $1.16 \pm 0.02$ \\
\hline 9 & J155019 & 495.44 & 135.39 & 103.95 & $1.14 \pm 0.06$ & $0.88 \pm 0.05$ & $1.19 \pm 0.02$ \\
\hline
\end{tabular}

Note. (1) Galaxy identification in the paper; (2) galaxy name; (3) continuum bandwidth (in $\AA$ ); (4) [N III]+H $\alpha$ filter bandwidth (in $\AA$ ); (5) [O III] filter bandwidth (in $\AA$ ); (6) continuum scale factor used for [O III]; (7) continuum scale factor used for [N II] $+\mathrm{H} \alpha$; (8) correction factor for the partial coverage of the [O III] $\lambda 4959$ line by the [O III] filter bandpass.

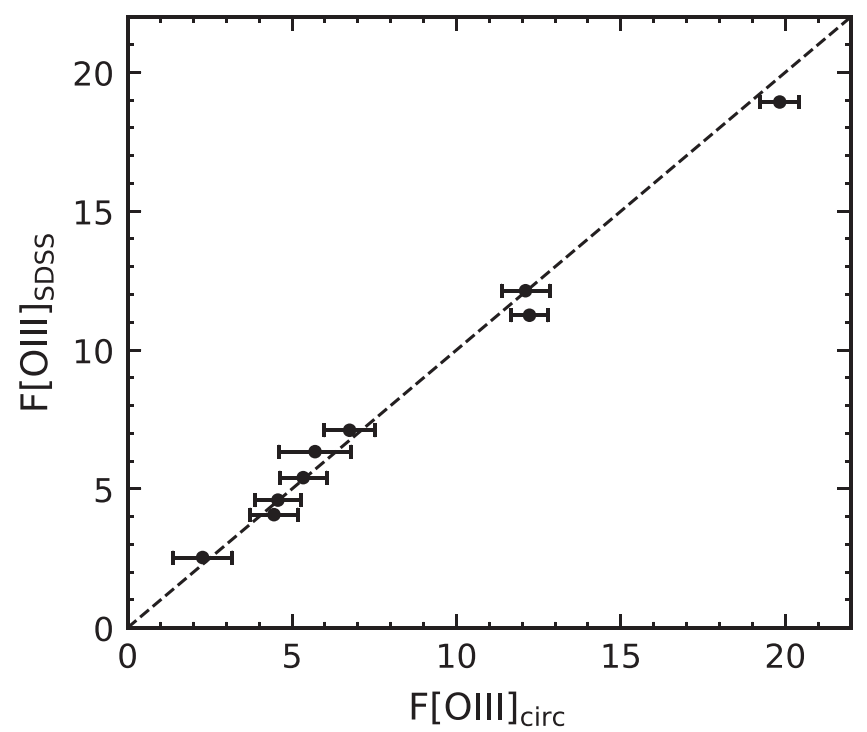

Figure 2. Relation between the flux $F[\mathrm{O} \mathrm{III}]_{\mathrm{SDSS}}$, obtained from the SDSS spectra, and $F[\mathrm{O} \mathrm{III}]_{\text {circ }}$, obtained from a circular aperture of $3^{\prime \prime}$ in our $H S T$ [O III] images. The dashed line corresponds to unity.

\section{Results}

As we have used the SDSS spectra to correct our continuum images from the contribution of the emission lines, as well as to correct for the usually partial coverage of the [O III] $\lambda 4959$ line emission, we begin by presenting below the results of the measurements of the SDSS integrated spectra (corresponding to an aperture of $3^{\prime \prime}$ diameter).

\subsection{SDSS Spectra Emission-line Fluxes}

The emission lines of the SDSS spectra were fitted using fourth-order Gauss-Hermite polynomials. In the case of the [O III] $\lambda 5007$ and $\lambda 4959$ emission lines, the same radial velocity and velocity dispersion were adopted, as well as the same $h_{3}$ and $h_{4}$ coefficients, and the two lines were constrained to have a flux ratio $F[\mathrm{O}$ III $] \quad \lambda 5007 / F[\mathrm{O}$ III $] \quad \lambda 4959=3$ (Osterbrock \& Ferland 2006). When one component was not enough to fit the line profile, additional components were included (keeping the same constraints as above between them). The fits were obtained via $\chi^{2}$ minimization using the SLSQP method available at the Python library SciPy. The same procedure was adopted in the fit of the [N II] doublet lines, and $\mathrm{H} \beta$ was fitted using one or more Gauss-Hermite profiles.

Objects 4,5 , and 8 show a broad base in the $[\mathrm{N} \mathrm{III}+\mathrm{H} \alpha$ emission lines, which could indicate the presence of a broad $\mathrm{H} \alpha$ component. However, we were able to fit these profiles similarly well without the need of a broad $\mathrm{H} \alpha$ component by assuming that both the $\mathrm{H} \alpha$ and $[\mathrm{N}$ II] profiles match that of [O III] $\lambda 5007$ in velocity space, with the broad base being a consequence of the merging of wings in the narrow emission lines. We opted for adopting this solution as the most physically compelling, even though a solution including a broad $\mathrm{H} \alpha$ component cannot be discarded. The total fluxes and widths of the emission lines in the SDSS spectra are displayed in Table 4. Uncertainties in the fit of the [O III] $\lambda 5007$ emission line are always below $2.5 \%$, while for the fainter lines, uncertainties reach at most $5 \%$.

We also include, in column 9 of Table 4, the value of the ratio $\eta=\mathrm{H} \alpha /([\mathrm{N} \mathrm{II}]+\mathrm{H} \alpha)$ obtained from the SDSS spectra that we have used to estimate the contribution of $\mathrm{H} \alpha$ to the narrowband HST images that contain both the $\mathrm{H} \alpha$ and [N II] emission lines. In the cases of the galaxies for which we could not obtain $\eta$ from the SDSS spectra (because these lines are beyond the observed spectral range), we have used the average of the values obtained for the other galaxies, and we list these values in bold in the table.

In the last column of Table 4 , we list the velocity $v_{80}=W_{80} /$ 1.3 , where $W_{80}$ is the width of the [O III] $\lambda 5007$ emission-line profile that comprises $80 \%$ of the line flux. This will be used in Section 5.5 as an estimate of the velocity of the gas outflow in the NLR, as it probes the highest-velocity gas that contributes to the emission in the profile wings.

\subsection{HST Images}

The corrected continuum and emission-line images are shown in Figures 3-11. In the top left panel, we show the continuum images; in the top right, the continuum-subtracted [O III] images; in the bottom right, the continuum-subtracted $\mathrm{H} \alpha+[\mathrm{N}$ II] images; and in the bottom left, an excitation map obtained as the ratio between the $[\mathrm{O}$ III] and $[\mathrm{N} \mathrm{II]}+\mathrm{H} \alpha$ images. The images have been multiplied by the bandwidth, and [O III] refers to the sum of the $\lambda 4959$ and $\lambda 5007$ emission lines. The white bar corresponds to $1^{\prime \prime}$ and shows the scale of the images in kpc. The black cross marks the position of the galaxy 
Table 4

SDSS Spectra Measurements

\begin{tabular}{|c|c|c|c|c|c|c|c|c|c|}
\hline $\begin{array}{l}\text { ID } \\
\text { (1) }\end{array}$ & $\begin{array}{l}\text { Name } \\
(2)\end{array}$ & $\begin{array}{c}F(\mathrm{H} \beta) \\
(3)\end{array}$ & $\begin{array}{c}F[\mathrm{O} \text { III }] \\
(4)\end{array}$ & $\begin{array}{c}F(\mathrm{H} \alpha) \\
\quad(5)\end{array}$ & $\begin{array}{c}F[\mathrm{~N} \mathrm{III}]_{\lambda 6584} \\
(6)\end{array}$ & $\begin{array}{c}\left.W_{[\mathrm{O}} \mathrm{III}\right] \\
\text { (7) }\end{array}$ & $\begin{array}{c}W_{\mathrm{H} \alpha} \\
(8)\end{array}$ & $\begin{array}{c}\eta \\
(9)\end{array}$ & $\begin{array}{l}v_{80} \\
(10)\end{array}$ \\
\hline 1 & J082313 & 0.290 & 4.595 & $\ldots$ & $\ldots$ & 375 & $\ldots$ & 0.571 & 411 \\
\hline 2 & J084135 & 0.761 & 12.135 & 3.296 & 1.081 & 396 & 429 & 0.696 & 336 \\
\hline 4 & J094521 & 1.335 & 18.93 & 5.186 & 4.947 & 456 & 467 & 0.440 & 896 \\
\hline 5 & J110952 & 0.342 & 6.34 & 1.920 & 2.207 & 1014 & 570 & 0.395 & 1324 \\
\hline 6 & $\mathrm{~J} 113710$ & 0.268 & 4.071 & 0.922 & 0.343 & 730 & 785 & 0.668 & 715 \\
\hline 9 & $\mathrm{~J} 155019$ & 1.193 & 11.252 & 4.784 & 1.122 & 355 & 366 & 0.734 & 333 \\
\hline
\end{tabular}

Note. (1) Galaxy identification in the paper. (2) Galaxy name. (3)-(6) Fluxes (in units of $10^{-14} \mathrm{erg} \mathrm{s}^{-1}$ ); in the case of [O III], it is the sum of the $\lambda \lambda 4959$, 5007 emission lines. (7)-(8) FWHM of the emission lines in units of $\mathrm{km} \mathrm{s}^{-1}$. (9) Ratio $\eta=\mathrm{H} \alpha /([\mathrm{N} \mathrm{II}]+\mathrm{H} \alpha)$, where the bold highlight indicates that the line was missing in the SDSS spectra and the value thus corresponds to the average of the ratios obtained for the other galaxies. (10) $v_{80}=W_{80} / 1.3$, where $W_{80}$ (in km s${ }^{-1}$ ) is the width of [O III] $\lambda 5007$ at a height corresponding to $80 \%$ of the integrated line flux.

nucleus, adopted as corresponding to the peak of the continuum flux.

The flux levels shown in the [O III] and [N II] $+\mathrm{H} \alpha$ images of Figures 3-11 range from $1 \sigma_{\text {sky }}$ to the maximum flux value in the galaxy image $\left(F_{\max }\right)$. Four equally spaced contours, ranging from $3 \sigma_{\text {sky }}$ to $F_{\text {max }}$, have been overplotted on these images. The contours were obtained after smoothing the images by a Gaussian filter with 1 pixel standard deviation.

In the case of the excitation maps, only pixels for which both images have values higher then $3 \sigma_{\text {sky }}$ were considered. The values of the remaining pixels were set to zero. We have introduced a contour in these maps to highlight regions (inside the contour) with the highest excitation values, typical of Sy galaxies, while lower values are typical of LINERs. This level was chosen at [O III] $\lambda 5007 / \mathrm{H} \beta=5.25$, and the conversion to $[\mathrm{O} \mathrm{III}] /([\mathrm{N} \mathrm{II}]+\mathrm{H} \alpha)$ was calculated using the corresponding fluxes of $[\mathrm{N} \mathrm{II}]+\mathrm{H} \alpha$ in the SDSS spectra (further discussed in Section 4.3). Dashed contours were used for galaxies for which the $[\mathrm{N} \mathrm{II}] / \mathrm{H} \alpha$ ratio could not be measured because these lines are beyond the spectral range of the SDSS spectrum and have thus been estimated using average data from other galaxies.

\subsubsection{Continuum}

Although the continuum images have been corrected for the average contamination of the emission lines via the $S_{\mathrm{C},[\mathrm{O} \mathrm{III}]}$ and $S_{\mathrm{C},([\mathrm{N} \mathrm{II}]+\mathrm{H} \alpha)}$ scale factors, the images in the continuum show some features that may be due to the contamination from the emission lines, as discussed in Section 3. The objects showing the largest corrections ( $>20 \%$ of the continuum flux) due to emission-line contamination are targets 1, 7, 6, and 3 (from largest to lowest corrections). Below, we discuss each of these cases and the possible consequences for the origin of the features seen in the continuum.

Target 1 (Figure 3 ) shows in the continuum image a possible companion to the southeast, although its continuum flux is low, while in the [O III] image, its emission is much stronger, even after subtracting the continuum with a significant emission-line contribution. This feature is thus most probably a detached cloud of gas and not a companion galaxy. In the case of target 7 (Figure 9), the extended feature seen in the continuum image to the southeast is most probably also due to contamination from the $[\mathrm{O} \mathrm{III}]$ emission lines, as seen by the strong corresponding feature in the [O III] image. In the case of target 6 (Figure 8), the extended appearance in the continuum from the southeast to the northwest may have some contamination from the strong emission seen in the [O III] line image. A compact object to the northwest is faint in the emission-line images and is most probably a companion in this case. Finally, in the case of target 3 (Figure 5), the continuum shows what seems to be a second nucleus, as its brightness approaches that of the nucleus (adopted as the brightest peak). Due to the [O III] emission-line contamination in the continuum filter, one may think that this feature is due to this contamination. But, if this is the case, then the [O III] image should have a strong knot of emission at this location, even if there is some contamination from the line to the continuum. But what we see instead is a small depression in the emission-line images at this location, which implies that there is not much [O III] emission in this knot, and the feature in the continuum is most probably a secondary nucleus from a possible recent capture of a small galaxy.

In the other targets, where the contamination of the continuum by the emission lines is lower than $20 \%$, it can be concluded that target 2 is in obvious interaction with a companion; target 4 shows some asymmetry in the continuum but, other than that, no signature of ongoing interaction; target 5 is essentially round, with no signature of interaction in the continuum; target 8 seems to be an ongoing merger; and target 9 shows an object $2^{\prime \prime}$ ( $\approx 5 \mathrm{kpc}$ in the plane of the sky) to the south that seems to be a companion galaxy.

The contamination of the emission lines in the continuum images may also cause a small decrease in the flux in the [O III] narrowband images, as well as in the $\mathrm{H} \alpha+[\mathrm{N} \mathrm{II}]$ images in the case of target 5 , but, as the structure of the continuum image is in most cases more compact than that of the emission-line images, it does not affect the properties of the most extended gas, in particular, the measured extent of the ENLR, one of the main results of the present paper.

\subsubsection{Emission-line Images}

All galaxies show [O III] and $\mathrm{H} \alpha+[\mathrm{N} \mathrm{II}]$ extended emission up to several kpc in the plane of the sky. Elongated structures clearly defining ionization axes are observed for targets $1,2,4$, $6,7,8$, and 9 , supporting the presence of a collimating structure of the nuclear radiation. The only exceptions are targets 3 

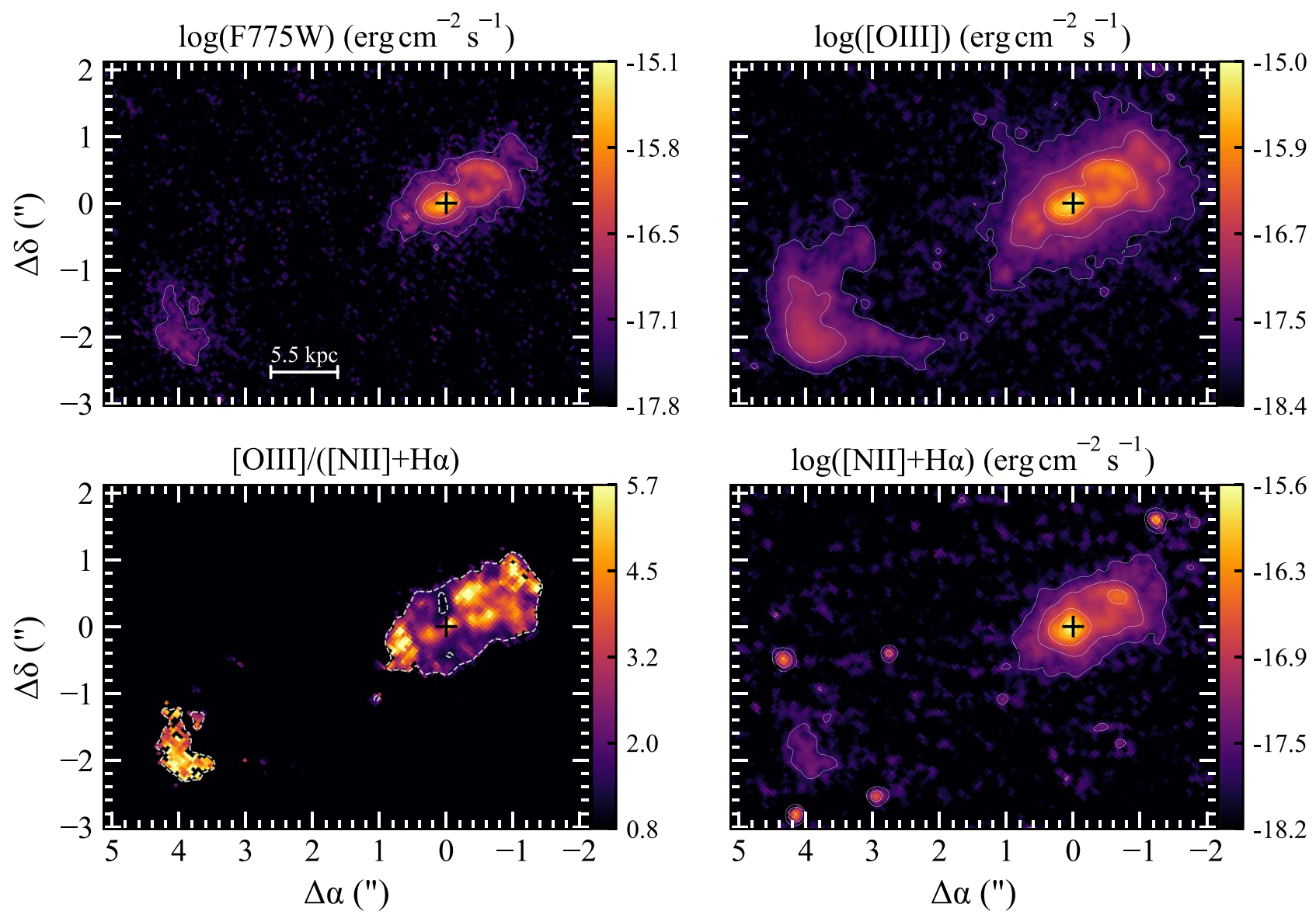

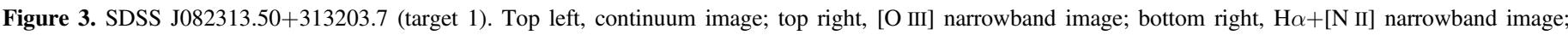

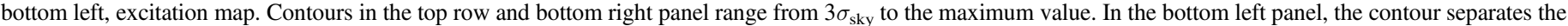

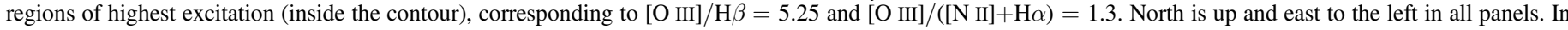

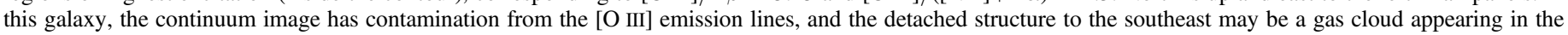
continuum due to this contamination (see the text).

and 5, which may be due to a more "face-on" orientation of the ionization axis.

\subsection{Excitation Maps}

The bottom left panels of Figures 3-11 show the excitation maps, obtained as the ratio between the [O III] and $[\mathrm{N} \mathrm{III}+\mathrm{H} \alpha$ images. As discussed above, we added a contour to these maps as a reference for the location of the regions with the highest excitation (inside the contours). The contour adopted to separate these values corresponds to [O III] $\lambda 5007 / \mathrm{H} \beta=5.25$, a value close to the separation between LINERs (below this value) and Sys (above this value) in the BPT $[\mathrm{O} \mathrm{III]}] / \mathrm{H} \beta$ versus $[\mathrm{N} \mathrm{II}] / \mathrm{H} \alpha$ diagnostic diagram (Baldwin et al. 1981; Veilleux \& Osterbrock 1987), considering values of $[\mathrm{N} \mathrm{II}] / \mathrm{H} \alpha \approx 0.3-1$ for our sample. The conversion of this $[\mathrm{O} \mathrm{III}] / \mathrm{H} \beta$ value to $[\mathrm{O} \mathrm{III}] /([\mathrm{N} \mathrm{II}]+\mathrm{H} \alpha)$ the actual ratio shown in our excitation maps-was obtained under the assumptions [O III] $\lambda 5007 /[\mathrm{O} \mathrm{III]} \lambda 4959=3$ and $\mathrm{H} \alpha / \mathrm{H} \beta=3$. This $\mathrm{H} \alpha / \mathrm{H} \beta$ ratio was adopted considering that, although its value is, on average, $\approx 4$ in the SDSS spectra, it should be dominated by the emission closest to the nucleus where there may be some reddening, while for most of the nebulae (farther from the nucleus), it should be closer to $\approx 3$. In any case, this contour is just a reference for the excitation level. In order to obtain the value of the $[\mathrm{O} \mathrm{III}] /([\mathrm{N} \mathrm{II}]+\mathrm{H} \alpha)$ ratio, we have used the $\eta$ value listed in Table 4. Considering the above, we have

$$
\frac{[\mathrm{O} \mathrm{III}]}{[\mathrm{N} \mathrm{II}]+\mathrm{H} \alpha}=\frac{4 \eta}{9}\left(\frac{[\mathrm{O} \mathrm{III}]_{\lambda 5007}}{\mathrm{H} \beta}\right) .
$$

These reference contours are shown as solid lines for galaxies with available $\eta$ values and dashed lines for galaxies with estimated $\eta$ values (shown in bold in Table 4). It is also important to point out that we are using a constant $\eta$ ratio throughout the ENLR, determined from the SDSS spectra, although this value most probably varies from pixel to pixel. Figures 3-11 show that the contours in most cases delineate a (patchy) approximately biconical structure for the highestionization regions (orange) surrounded by lower-ionization regions (purple). In the cases of targets 1 and 7, these contours seem to be beyond the region separating the highest from the lowest excitation, but the biconical structure is delineated by the orange regions surrounded by purple regions, with the separation between them appearing at higher line ratios than that of the adopted contour.

\subsection{1. $[O$ III $] \lambda 5007$ and $[O$ III $] / H \beta$ Spatial Profiles}

In order to better quantify the excitation along the ENLR, we have used the $\eta$ values listed in Table 4 to obtain the line-ratio values [O III] $\lambda 5007 / \mathrm{H} \beta$ (hereafter $[\mathrm{O} \mathrm{III}] / \mathrm{H} \beta$ ) and construct one-dimensional spatial profiles for the [O III] fluxes and 

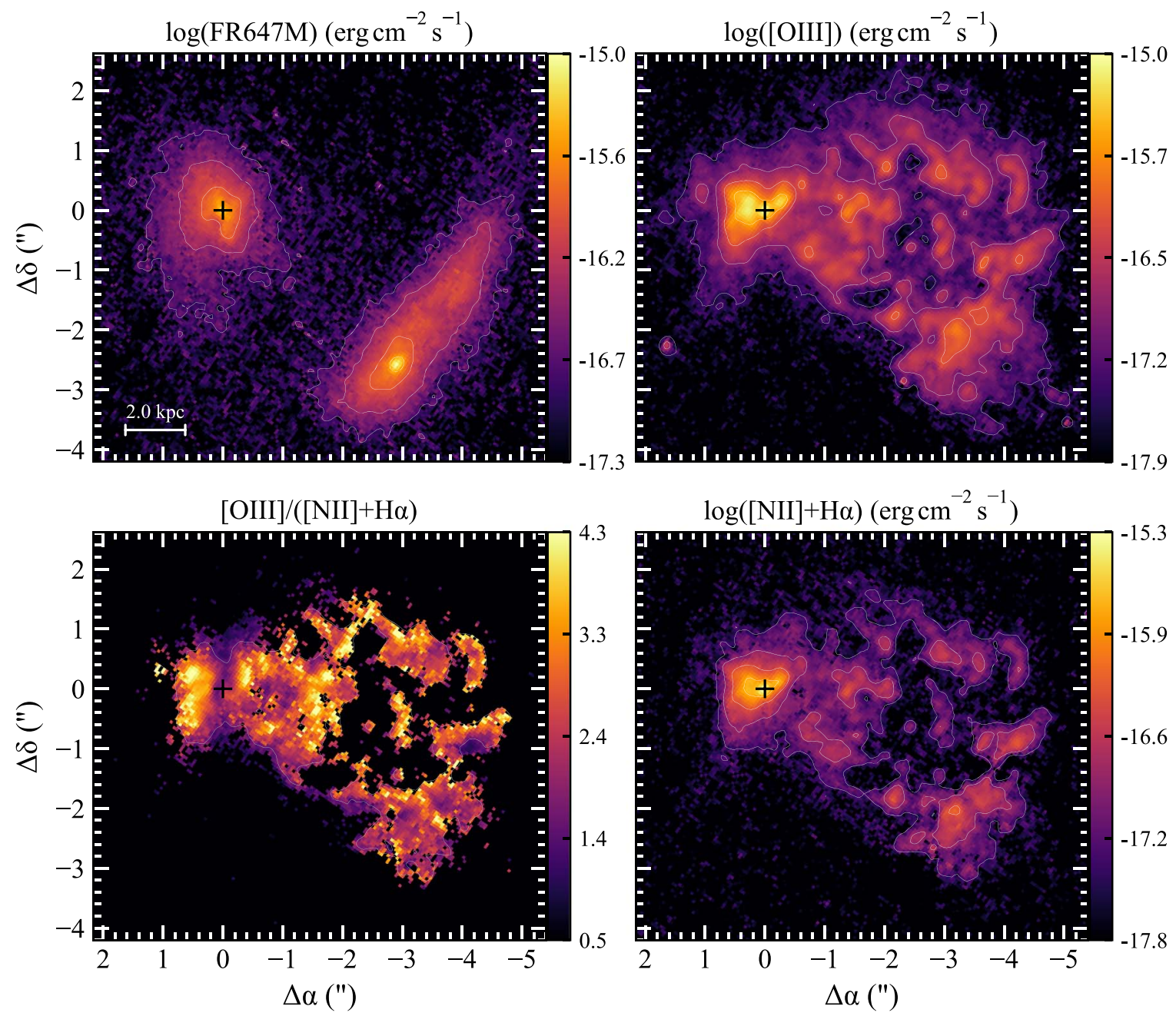

Figure 4. Same as Figure 3 but for SDSS J084135.04+010156.3 (target 2).

[O III] $/ \mathrm{H} \beta$ ratios through the nucleus along the ionization axis and perpendicular to it within pseudo-slits with a width corresponding to $0 ! .05$ ( $\approx 100-300 \mathrm{pc}$ at the galaxies).These one-dimensional profiles are shown in Figure 12. The [O III] profiles are only shown along the ionization axis.

\subsection{Extent of the ENLR}

We have measured the total extent of the ionized gas emission in the [O III] images along the ionization axis up to an emission level corresponding to the $3 \sigma_{\text {sky }}$ contour. The $\sigma_{\text {sky }}$ value of the [O III] image is listed in the last column of Table 5. The orientation of the ionization axis-also listed under PA in Table 5-was adopted as that corresponding to the longest axis of symmetry of the excitation maps in Figures 3-11. Figure 13 shows the adopted extents over the [O III] images for each galaxy. The uncertainty in this extent was estimated as the difference between the values measured using the contours at $2 \sigma_{\text {sky }}$ and $4 \sigma_{\text {sky. }}$.

The radius of the ENLR - which we hereafter call $R_{\text {maj }}$-was adopted to be half the value in kpc corresponding to the above angular extents (following Schmitt et al. 2003b), with an uncertainty propagated from those in the angular extent and corresponding galaxy scale. The $R_{\text {maj }}$ values are listed in Table 5 and range from 4 to $19 \mathrm{kpc}$.

\subsection{Emission-line Luminosities and Gas Masses}

The total emission-line luminosities $L[\mathrm{O}$ III] and $L([\mathrm{~N}$ II] $+\mathrm{H} \alpha)$ as derived from the images were obtained by integrating the flux values above $3 \sigma_{\text {sky }}$ using the distances listed in Table 1. The resulting values are listed in Table 5, where $L[\mathrm{O} \mathrm{IIII}]_{\lambda 5007}$ was obtained from the images after multiplying them by 0.75 in order to eliminate the contribution of the [O III] $\lambda 4959$ emission line.

\subsubsection{Total Ionized Gas Masses}

We have estimated the total ionized gas mass of the ENLR from $L([\mathrm{~N} \mathrm{II}]+\mathrm{H} \alpha$ ) for case B recombination (Osterbrock \& Ferland 2006) following Peterson (1997). The total luminosity of $\mathrm{H} \beta$, originating from clouds within a total volume $V_{c}$, is $L(\mathrm{H} \beta)=n_{e} n_{p} \alpha_{\mathrm{H} \beta}^{\mathrm{eff}} h \nu_{\mathrm{H} \beta} V_{c}$, where $\alpha_{\mathrm{H} \beta}^{\text {eff }}$ and $\nu_{\mathrm{H} \beta}$ are the effective recombination coefficient and the rest frequency of $\mathrm{H} \beta$, respectively, and $n_{e}$ and $n_{p}$ are the number densities of electrons and protons. We consider completely ionized hydrogen clouds, thus $n_{e} \approx n_{p}$. The $\mathrm{H} \alpha$ luminosity can be written as $L(\mathrm{H} \alpha)=\left(j_{\mathrm{H} \alpha} / j_{\mathrm{H} \beta}\right) L(\mathrm{H} \beta)$, where $j_{\mathrm{H} \alpha} / j_{\mathrm{H} \beta}$ is the ratio between $\mathrm{H} \alpha$ and $\mathrm{H} \beta$ emissivities. Assuming $L(\mathrm{H} \alpha)=\eta, L$ $([\mathrm{N} \mathrm{II}+\mathrm{H} \alpha])$, and the same density for all clouds $-n_{p} m_{p}$, where $m_{p}$ is the proton mass-the total ionized mass is 

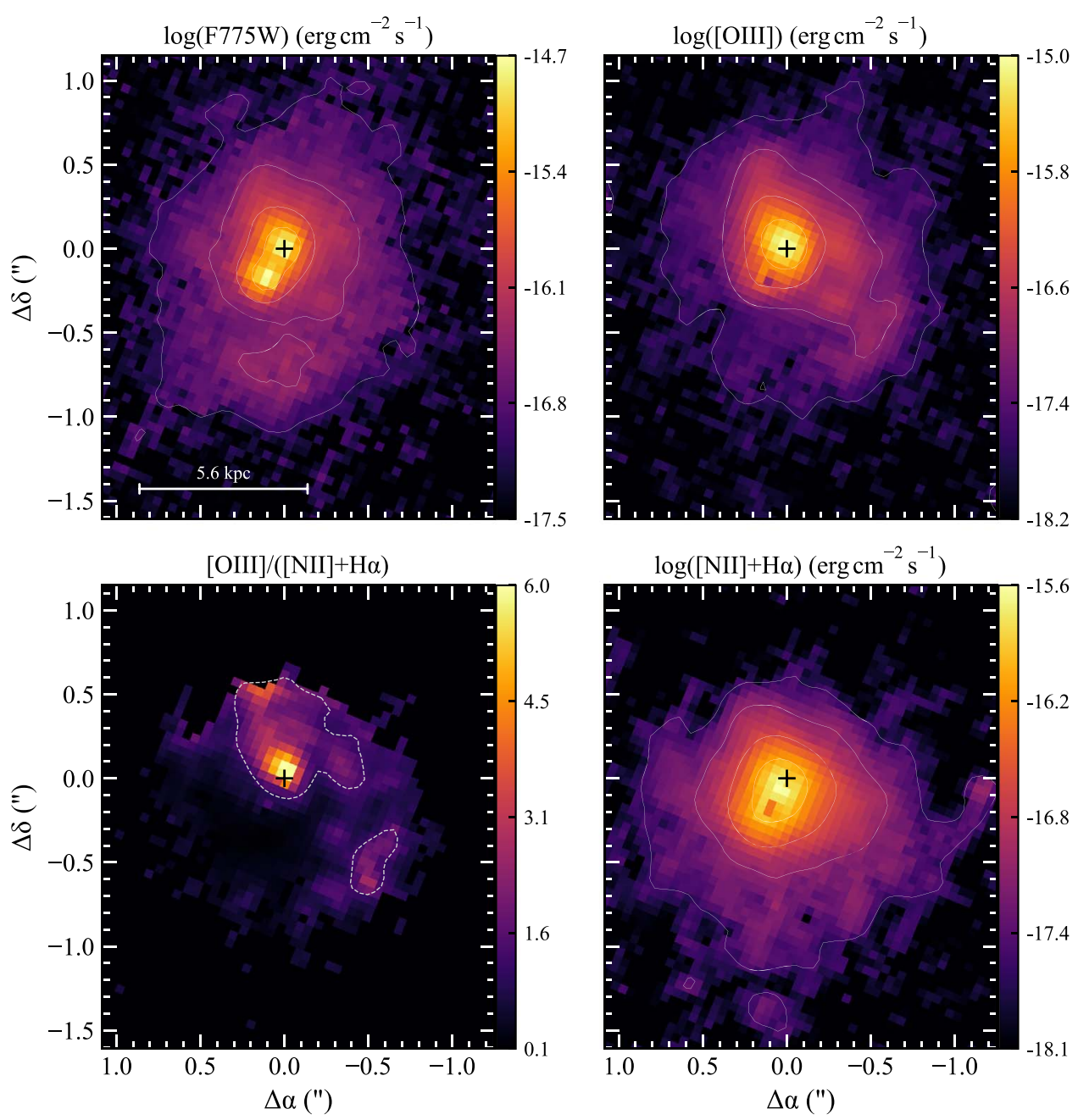

Figure 5. Same as Figure 3 but for SDSS J085829.58+441734.7 (target 3). In this galaxy, the continuum image has contamination from the [O III] emission lines, but the secondary nucleus seen in the continuum image is not due to this contamination (see the text).

$M_{\mathrm{ENLR}}=\left(n_{p} m_{p}\right) V_{c}$. Using the equations above,

$$
\begin{gathered}
M_{\mathrm{ENLR}}=\frac{m_{p} \eta L([\mathrm{~N} \mathrm{II}]+\mathrm{H} \alpha)}{n_{e}\left(j_{\mathrm{H} \alpha} / j_{\mathrm{h} \beta}\right) \alpha_{\mathrm{H} \beta}^{\mathrm{eff}} h \nu_{\mathrm{H} \beta}} \\
=0.238 \eta L_{42}([\mathrm{~N} \mathrm{II}]+\mathrm{H} \alpha) M_{\odot},
\end{gathered}
$$

where $L_{42}([\mathrm{~N} \mathrm{II}]+\mathrm{H} \alpha)$ is in units of $10^{42} \mathrm{erg} \mathrm{s}^{-1}$. As we do not have resolved density values from our images, we have adopted a constant density of $100 \mathrm{~cm}^{-3}$ throughout the ENLR as a compromise. The value of the density should be higher in the inner kpc or so (thus, we are overestimating the mass for this region) but lower for the outer kpc (thus, we are underestimating the mass for the external regions). The values for the emission coefficient and recombination ratio have been obtained from Osterbrock \& Ferland (2006) for $n_{e}=$ $100 \mathrm{~cm}^{-3}$ and $T=10^{4} \mathrm{~K}$. The resulting $M_{\mathrm{ENLR}}$ masses are shown in column 9 of Table 5.

\subsubsection{Ionized Gas Mass Profiles}

We can use the ionized gas masses per pixel calculated as above to obtain the ionized gas surface mass densities. As we have adopted a constant gas density, the corresponding maps are very similar to those of the $\mathrm{H} \alpha+[\mathrm{N} \mathrm{III}$. We instead show a one-dimensional profile for the mass distribution binning the data within square regions corresponding to $1 \mathrm{kpc}^{2}$ at the galaxies. We then built a cumulative mass profile along the ionization axis by adding all masses perpendicular to this axis within each kpc. These one-dimensional cumulative mass distributions, shown in Figure 14, present a "central peak" that reaches values of $10^{7.5} M_{\odot}$ in the inner kpc and decreases to $10^{5} M_{\odot}$ at about $5 \mathrm{kpc}$ from the nucleus for targets $4,5,7$, and 9 , which show an approximately symmetric mass distribution relative to the nucleus. Asymmetric (more extended to one side) and more extended mass distributions are observed for the remaining objects, whose values of cumulative masses within each kpc along the ionization axis range from $\sim 10^{6}$ to $\sim 10^{7} M_{\odot}$ at distances between 10 and $20 \mathrm{kpc}$. Targets 1,6 , and 8 show the most extended mass distributions. All objects with asymmetric mass profiles correspond to galaxies with signatures of interactions, the only exception being target 9 , which, although seeming to have a symmetric mass profile, shows a companion.

\section{Discussion}

One recent development in the study of AGNs was the realization that AGNs flicker on scales $\leqslant 10^{5} \mathrm{yr}$ (e.g., Novak 

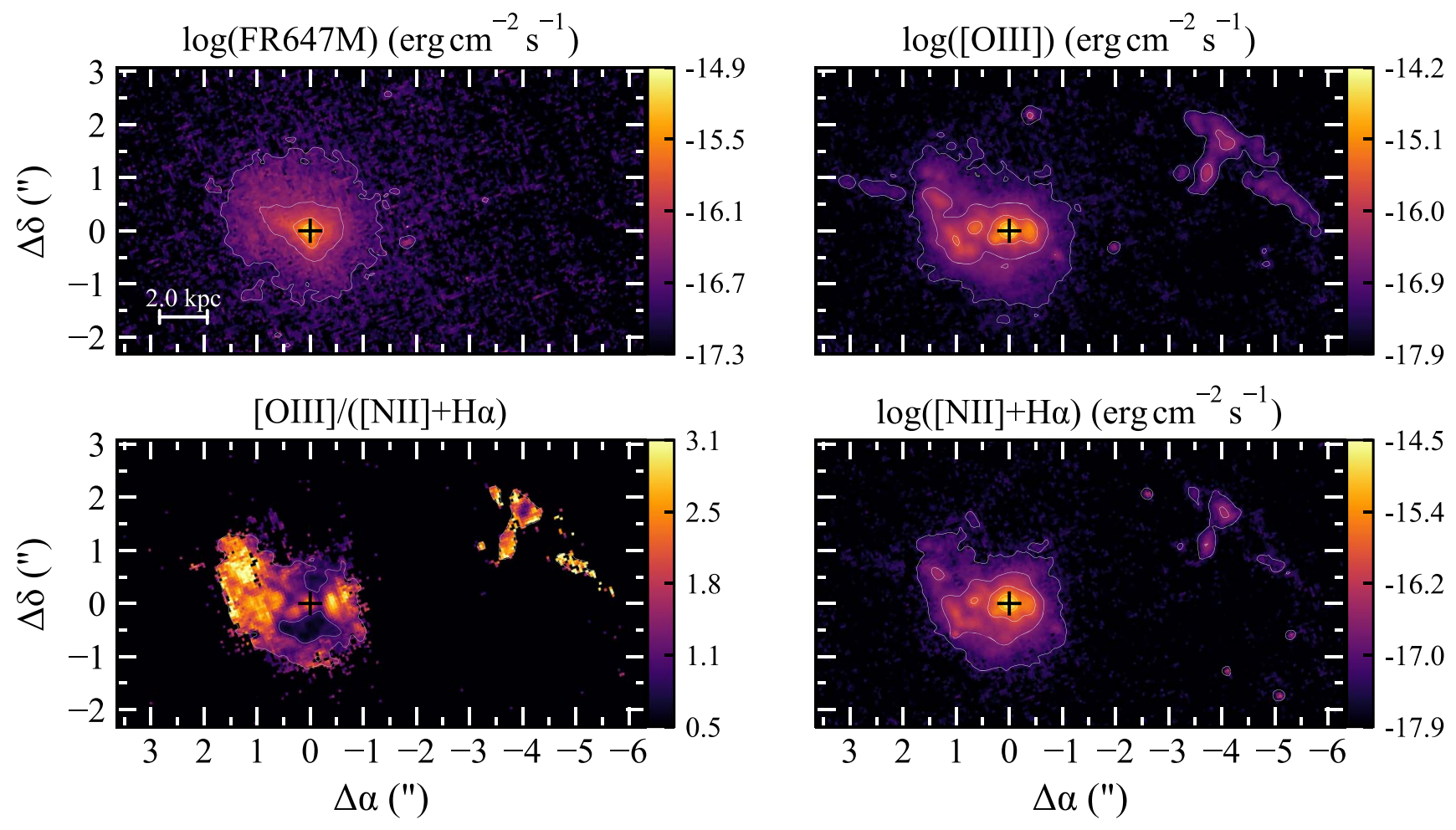

Figure 6. Same as Figure 3 but for SDSS J094521.34+173753.3 (target 4).
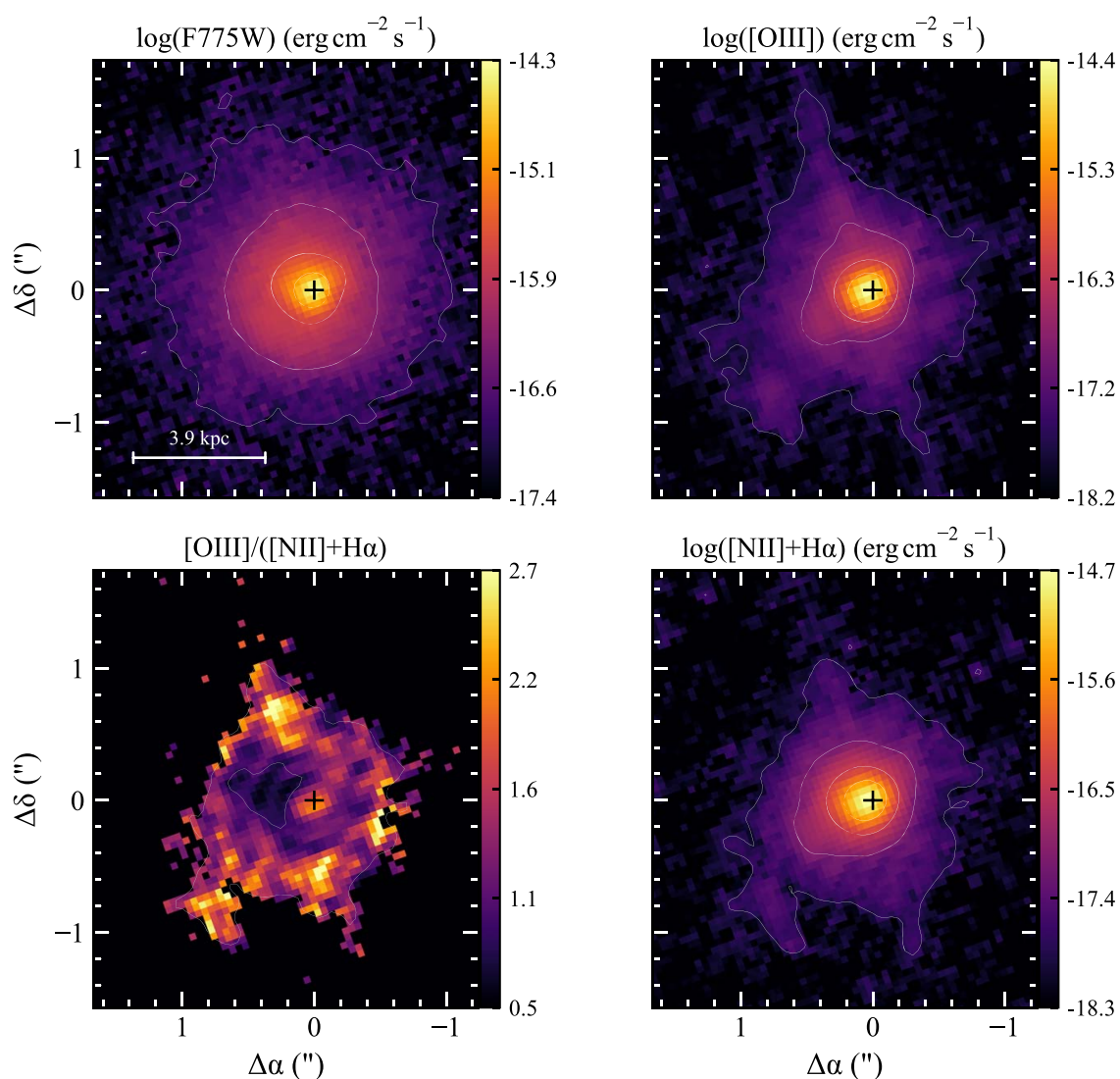

Figure 7. Same as Figure 3 but for SDSS J110952.82+423315.6 (target 5).

et al. 2011; Hickox et al. 2014), which is supported by observations of even our home Galaxy by the discovery of the "Fermi bubbles" in the vicinity of the Galactic center
(Su et al. 2010). Further evidence of this past activity of the galaxy center has also been seen in the form of a "fossil imprint" of a past powerful flare (1-3 Myr ago) on the 

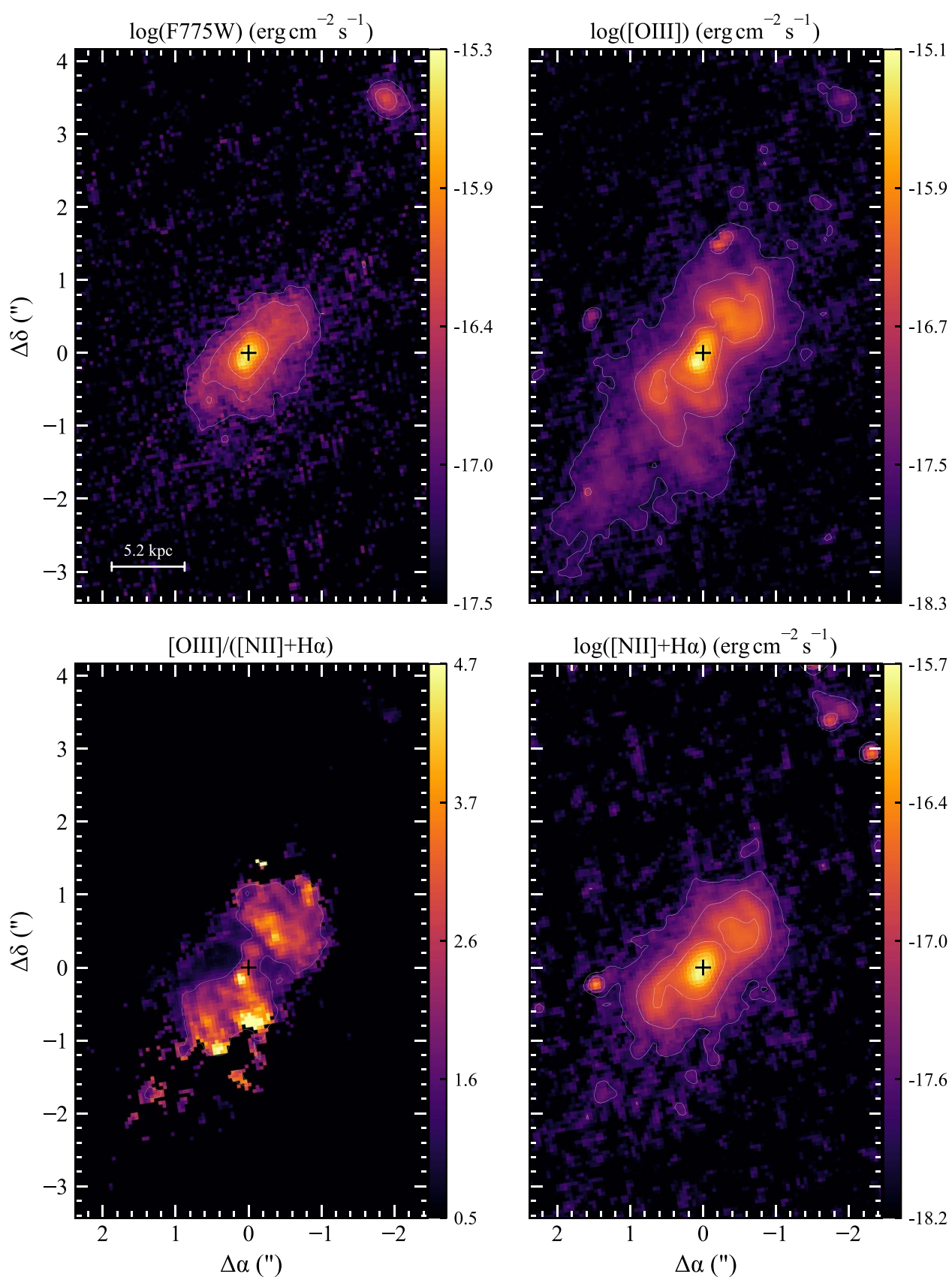

Figure 8. Same as Figure 3 but for SDSS J113710.77+573158.7 (target 6). In this galaxy, the continuum image has contamination from the [O III] emission lines, but the detached structure to the northwest may be a companion, as it does not appear in the emission-line images (see the text).

Magellanic Stream at a distance of $50-100 \mathrm{kpc}$ from the galactic center (Bland-Hawthorn et al. 2013). In addition, two absorbing structures with velocities of -235 and $+250 \mathrm{~km} \mathrm{~s}^{-1}$ in the light of background quasars have been recently observed between the galactic nucleus and these ionized regions of the Magellanic Stream (Fox et al. 2015), in line with an origin in the front and back walls of a bipolar outflow due to this past nuclear activity.

In light of this known intermittency of the nuclear activity in galaxies, it is somewhat unexpected that the line emission from the ENLR is as continuous as observed in our sample. Eventual patchiness can be attributed to irregularities in the mass distribution, but there are no clear signatures of light echoes. An explanation for this has been previously proposed (Crenshaw et al. 2003; Sharp \& Bland-Hawthorn 2010): due to the low gas density of the most external regions of the ENLR $\left(\leqslant 1 \mathrm{~cm}^{-3}\right)$, the recombination time becomes of the order of the flicker time of $\approx 10^{5-6} \mathrm{yr}$. In any case, a time-dependent analysis of the AGN photoionization is something we plan to do in the near future (e.g., Bland-Hawthorn et al. 2013) using resolved spectroscopy that is being acquired for the galaxies of our sample.

\subsection{The Ionization Cones}

Inspection of Figures 3-11 shows that most narrowband images present an elongated morphology indicating collimation of the ionizing radiation. The exceptions are targets 3 and 5, which show a more compact gas distribution. One possibility is that the orientation of the ionization axis is closer to "face-on" in these cases. In the case of target 5, there is evidence that this is indeed 

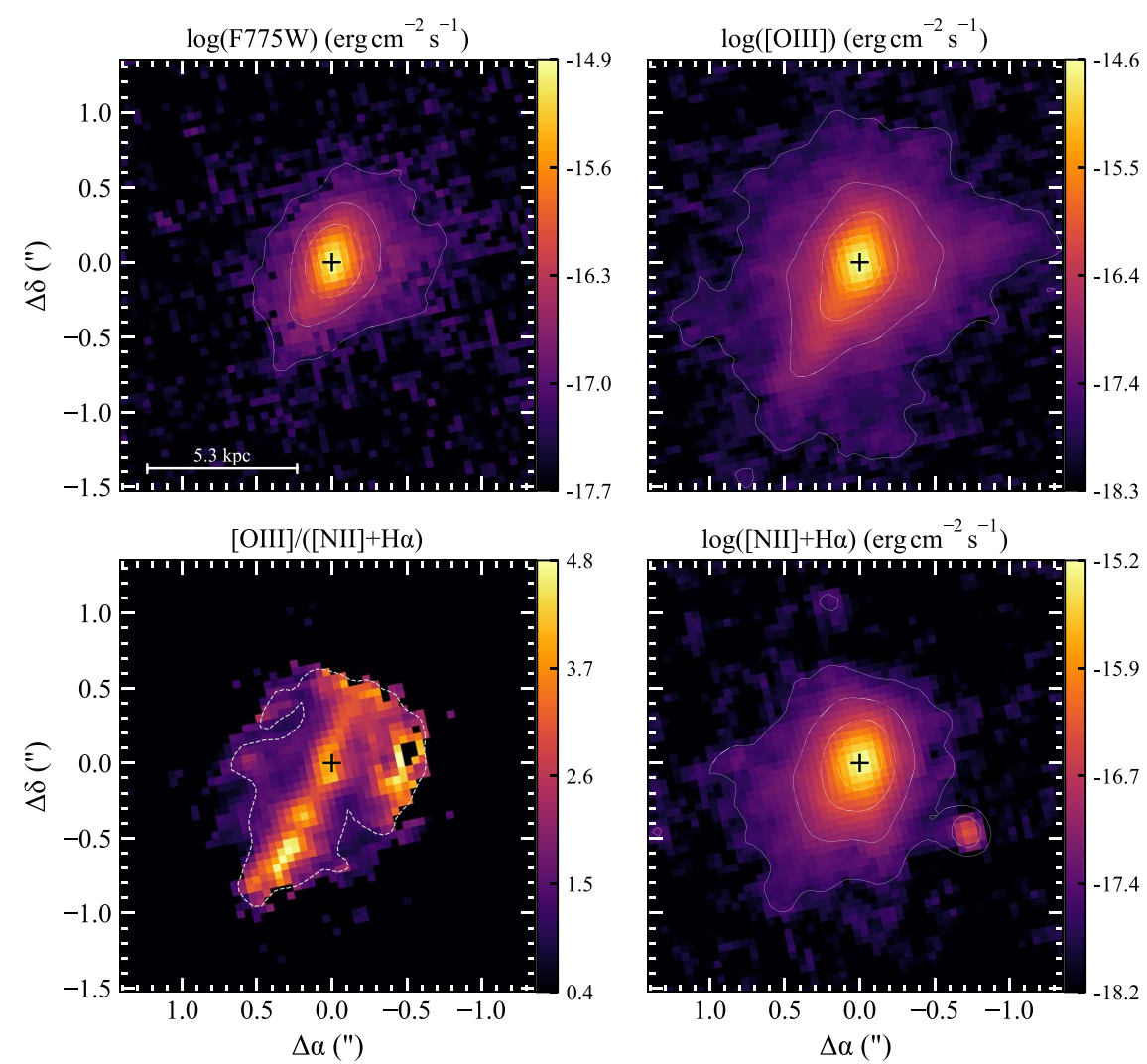

Figure 9. Same as Figure 3 but for SDSS J123006.79+394319.3 (target 7). In this galaxy, the continuum image has contamination from the [O III] emission lines, and the elongation to the southeast may be due to this contamination (see the text).
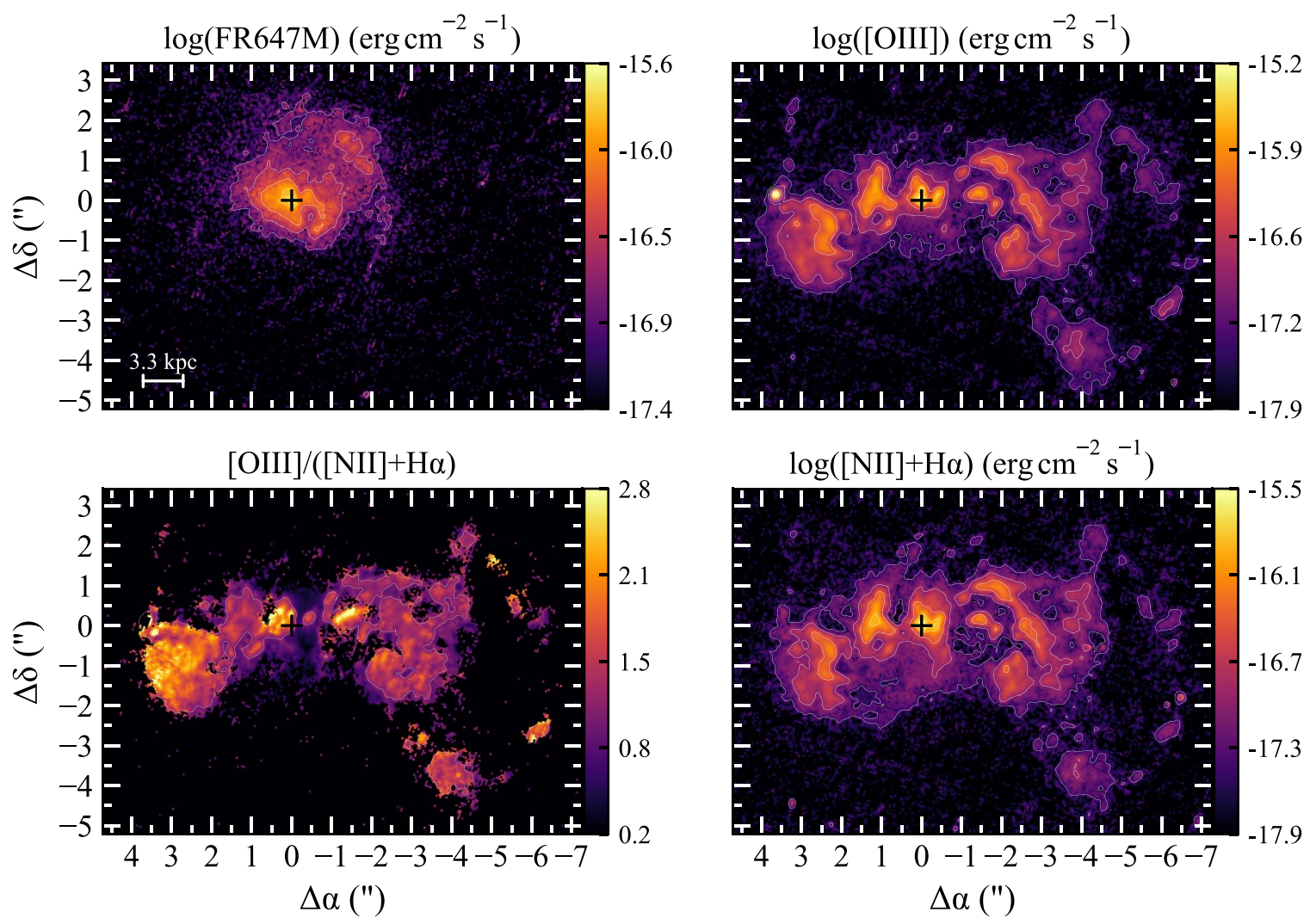

Figure 10. Same as Figure 3 but for SDSS J135251.21+654113.2 (target 8). 

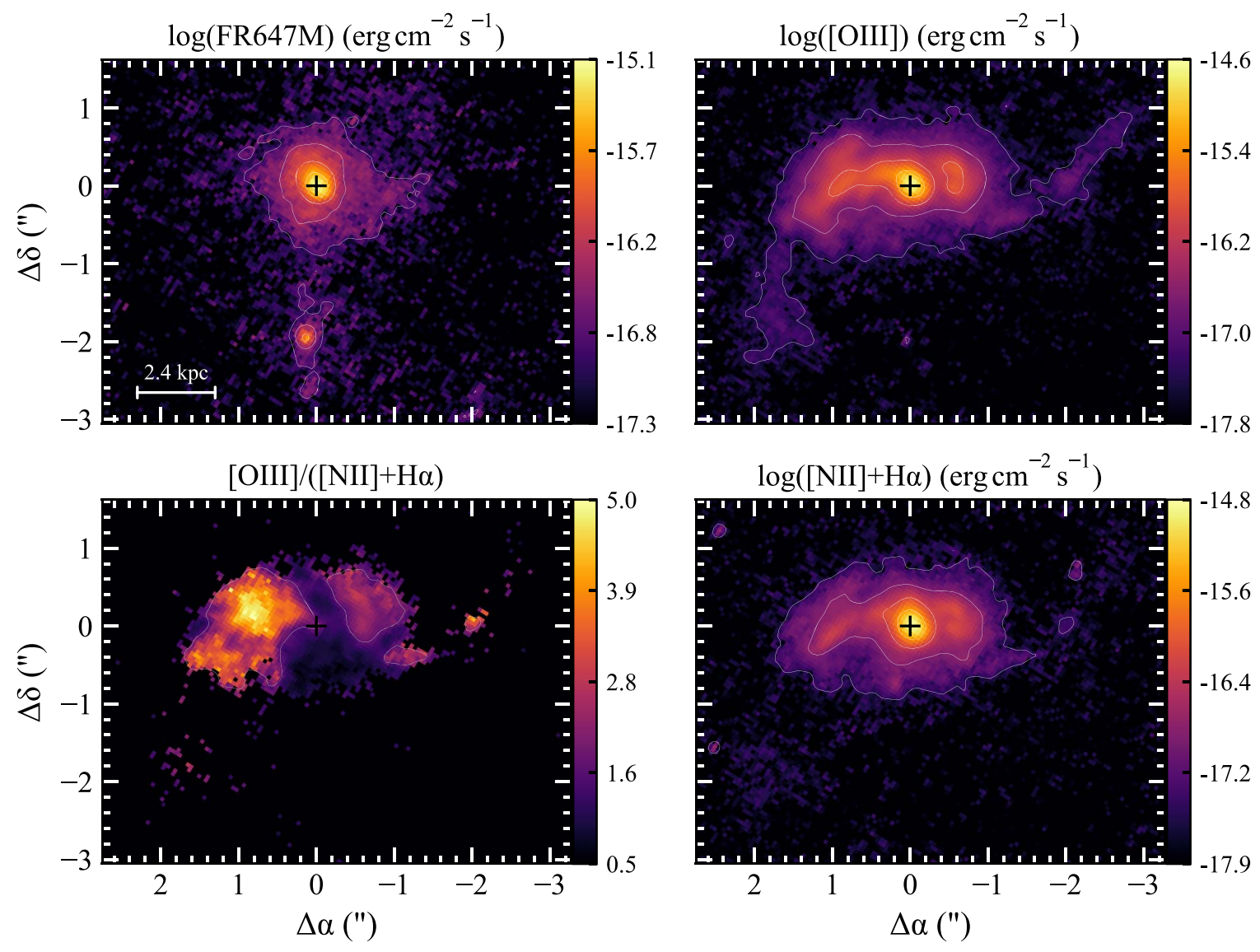

Figure 11. Same as Figure 3 but for SDSS J155019.95+243238.7 (target 9).

the case. This target shows the broader base in the $\mathrm{H} \alpha+[\mathrm{N}$ II $]$ of the SDSS spectrum. Although we favored a fit without a broad $\mathrm{H} \alpha$ component, the data are also consistent with the presence of such a component within the fitting uncertainties, in which case the observation of a Sy 1 nucleus would be consistent with a more pole-on visualization of the AGN, explaining the "rounder" ENLR in this galaxy. A "pole-on" view of the ENLR and the possible associated outflow is also consistent with the high value of $v_{80} \sim 1300 \mathrm{~km} \mathrm{~s}^{-1}$ listed in Table 4 for this galaxy, possibly due to a high outflow velocity component, as expected if the direction of the outflow is close to the line of sight.

The excitation maps in Figures 3-11 reveal ionization bicones in six of our nine targets, namely targets $1,2,4,6,8$, and 9, with the highest excitation levels-with $[\mathrm{O} \mathrm{III}] /(\mathrm{H} \alpha$ $+[\mathrm{N}$ II] $)$ in the range $\approx 4-6$, shown as orange in the figurescloser to their central axes, surrounded by lower excitation levels with $[\mathrm{O} \mathrm{III}] /(\mathrm{H} \alpha+[\mathrm{N} I \mathrm{II}]) \leqslant 1$, shown as purple in the figures. Lower excitation is also observed surrounding the apex of the cones, perpendicular to the ionization axis, supporting an obscuration of the nucleus along our line of sight due to a collimating structure-presumably the dusty torus postulated in the unified model (Antonucci 1993).

Our results thus do not support the disappearance of the torus for high-luminosity AGNs, as postulated by some studies (e.g., Elitzur et al. 2014), at least up to luminosities of $L[\mathrm{O} \mathrm{III]}=$ $2.5 \times 10^{43} \mathrm{erg} \mathrm{s}^{-1}$ (the highest AGN luminosity in our sample). Although two of our targets show a "rounder" morphology for the ENLR, this can be attributed to orientation effects, at least in the case of target 5 , as discussed above. We thus argue that the preferred rounder morphologies for the
ENLR found in previous ground-based studies (e.g., Liu et al. 2013) may be due to the lack of angular resolution to resolve the biconical morphology. For example, in the case of target 2 (Figure 4), the region with low excitation perpendicular to the ionization axis that defines the biconical morphology has an angular width of only $\approx 0$ ! 2 , which is not resolved by previous ground-based studies without adaptive optics. The spatial resolution of the HST observations has been fundamental in revealing the presence of the torus at these high luminosities.

In order to further explore the ionization cones, we now discuss spatial one-dimensional line-ratio profiles obtained through the ENLR.

\subsection{1. [O III]/Hß Spatial Profiles}

Figure 12 shows spatial profiles of $[\mathrm{O} \mathrm{III}] / \mathrm{H} \beta$ passing through the nucleus along the ionization axis (blue and turquoise symbols) and perpendicular to it (red and orange symbols) in logarithmic units. Also shown are the corresponding spatial profiles of the [O III] fluxes along the ionization axis (black and gray lines). A trend can readily be seen for the line ratios: while along the ionization axis (blue symbols), the values are the highest, in the range $0.9 \leqslant \log ([\mathrm{O}$ III $]) / \mathrm{H} \beta \leqslant$ 1.2 , corresponding to $8 \leqslant[\mathrm{O} \mathrm{III]} / \mathrm{H} \beta \leqslant 16$ along the perpendicular direction (the torus), they are lower, in the range $0.5 \leqslant$ $\log ([\mathrm{O} \mathrm{III}]) / \mathrm{H} \beta \leqslant 0.8$, corresponding to $3 \leqslant[\mathrm{O} \mathrm{III}] / \mathrm{H} \beta \leqslant 7$.

Although we defer a more in-depth analysis of the excitation structure of the ENLR to future studies via resolved spectroscopy of our targets, preliminary results can be obtained by comparing the typical line ratios listed above for the 

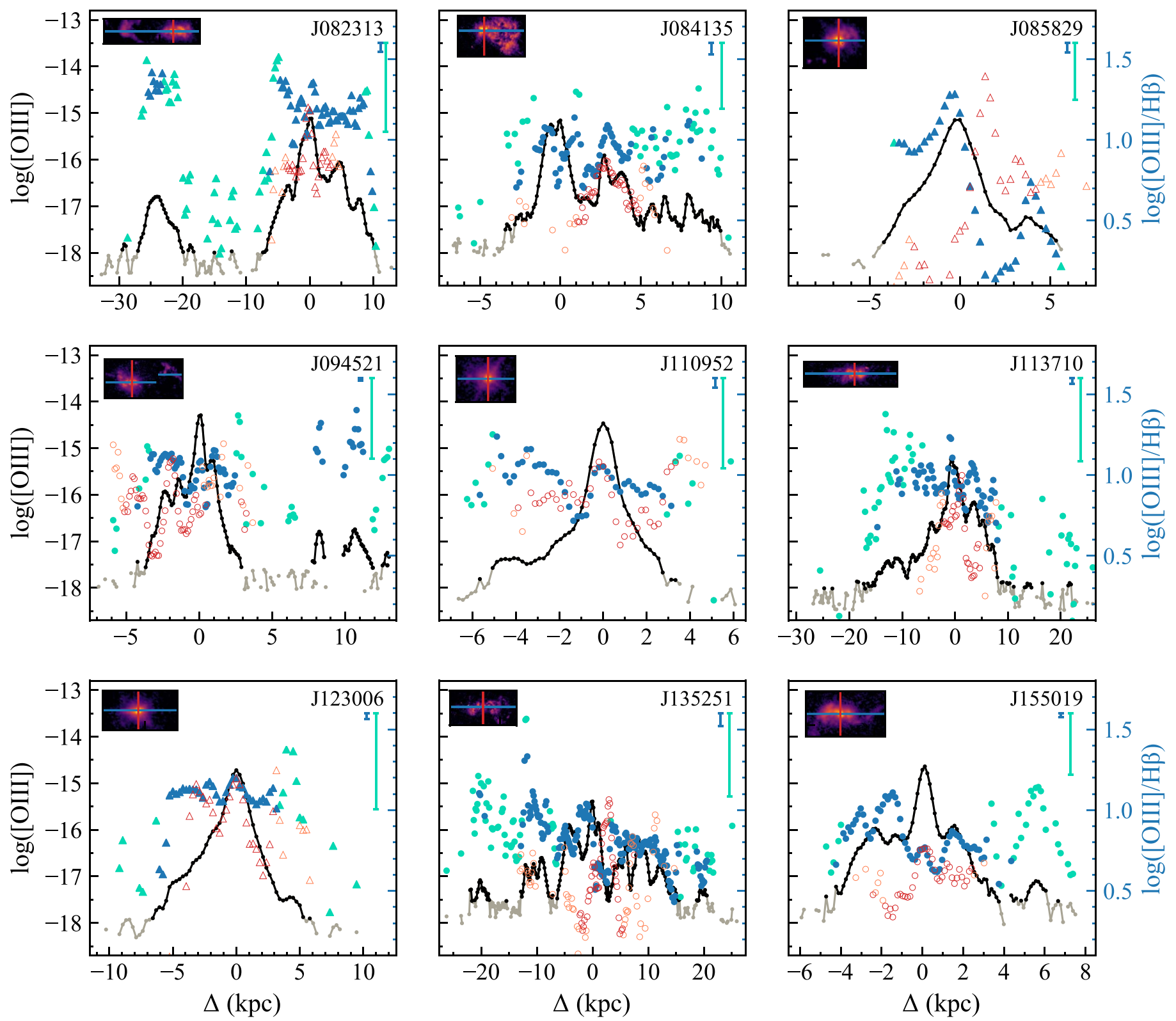

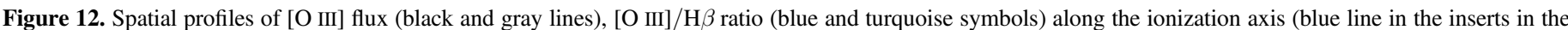

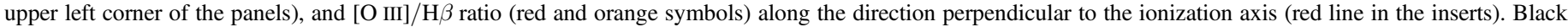

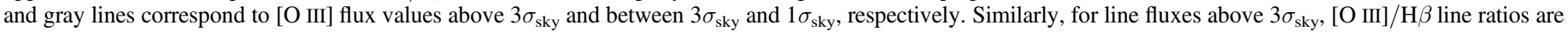

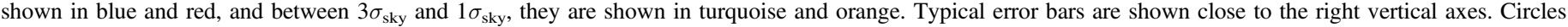

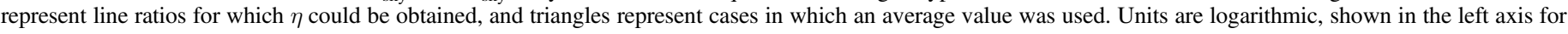
the [O III] fluxes and the right axis for the line ratios.

ionization cone and the values perpendicular to it with those of photoionization models. We have used as reference the work of Kreimeyer \& Veilleux (2013) and their photoionization models constructed to reproduce the line ratios of the very extended emission nebula around the quasar MR 2251-178, which shows an ionization cone extending to $\approx 90 \mathrm{kpc}$ from the nucleus. Adopting the $[\mathrm{NII}] / \mathrm{H} \alpha$ ratios of our targets from the SDSS spectra-in the range $0.3-1$, thus $-0.5 \leqslant \log ([\mathrm{N}$ II $]) / \mathrm{H} \alpha) \leqslant 0$ -from Figure 3(d) of Kreimeyer \& Veilleux (2013; dust-free photoionization models) for the typical range of the line ratios along the direction of the torus in our images, the ionization parameter value is $\log (U) \approx-3$, while for the line ratios along the ionization cone, $\log (U) \approx-2$.
Considering that $U=Q /\left(4 \pi R^{2} n_{e} c^{2}\right)$, where $Q$ is the rate of ionizing photons from the AGN, $R$ is the distance from the nucleus, and $n_{e}$ is the electronic density, and that for the inner region of the cones and the perpendicular direction, $n_{e}$ and $R$ should be similar, the difference in $U$ is reflecting the difference in $Q$. This implies that the rate of ionizing photons escaping along the cones is $\approx 10$ times the rate escaping perpendicular to the cone, supporting the presence of an obscuring and collimating structure such as the torus.

Besides decreasing along the direction perpendicular to the ionization axis due to dilution of the radiation by the torus, the $[\mathrm{O} \mathrm{III}] / \mathrm{H} \beta$ ratios also decrease in certain regions due to other factors. In the case of target 3 , there is a sharp decrease at 
Table 5

Measurements from the HST Images

\begin{tabular}{|c|c|c|c|c|c|c|c|c|c|}
\hline $\begin{array}{l}\text { No. } \\
\text { (1) }\end{array}$ & $\begin{array}{l}\text { Name } \\
(2)\end{array}$ & $\begin{array}{c}F[\mathrm{O} \text { III }]_{\text {circ }} \\
\text { (3) }\end{array}$ & $\begin{array}{c}L[\mathrm{O} \mathrm{IIII}]_{\lambda 5007} \\
\quad(4)\end{array}$ & $\begin{array}{l}\text { PA } \\
(5)\end{array}$ & $\begin{array}{c}\theta \\
(6)\end{array}$ & $\begin{array}{l}R_{\text {maj }} \\
\text { (7) }\end{array}$ & $\begin{array}{c}L(\mathrm{H} \alpha+[\mathrm{N} \text { II }]) \\
(8)\end{array}$ & $\begin{array}{l}M_{\mathrm{ENLR}} \\
\quad(9)\end{array}$ & $\begin{array}{c}\sigma_{\text {sky, }[\mathrm{O} \text { mI] }} \\
\quad(10)\end{array}$ \\
\hline 1 & J082313 & $4.57 \pm 0.69$ & $25.0 \pm 4.3$ & 117 & $6.87 \pm 0.13$ & $18.76 \pm 0.93$ & $8.9 \pm 8.4$ & $1.2 \pm 1.2$ & 0.35 \\
\hline 2 & J084135 & $12.10 \pm 0.73$ & $5.37 \pm 0.65$ & 90 & $6.33 \pm 0.34$ & $6.18 \pm 0.45$ & $2.38 \pm 0.31$ & $0.398 \pm 0.052$ & 1.1 \\
\hline 4 & J094521 & $19.83 \pm 0.60$ & $6.47 \pm 0.61$ & 90 & $7.58 \pm 0.19$ & $8.41 \pm 0.47$ & $4.70 \pm 0.45$ & $0.496 \pm 0.048$ & 1.1 \\
\hline 5 & $\mathrm{~J} 110952$ & $5.7 \pm 1.1$ & $8.2 \pm 1.6$ & 142 & $2.07 \pm 0.14$ & $4.05 \pm 0.34$ & $7.4 \pm 2.0$ & $0.70 \pm 0.19$ & 0.56 \\
\hline 6 & J113710 & $4.45 \pm 0.72$ & $18.4 \pm 3.3$ & 146 & $4.75 \pm 0.45$ & $12.3 \pm 1.3$ & $9.8 \pm 5.8$ & $1.57 \pm 0.93$ & 0.45 \\
\hline 9 & J155019 & $12.22 \pm 0.55$ & $4.83 \pm 0.51$ & 98 & $4.99 \pm 0.69$ & $6.04 \pm 0.89$ & $3.37 \pm 0.37$ & $0.594 \pm 0.066$ & 1.2 \\
\hline
\end{tabular}

Note. (1) Identification number in the paper; (2) galaxy name; (3) [O III] flux measured in the HST image within an aperture of $3^{\prime \prime}$ (in units of $10^{-14} \mathrm{erg} \mathrm{s}^{-1}$ ); (4) total [O III] $\lambda 5007$ luminosity, integrated above $3 \sigma_{\text {sky }}$ (in units of $10^{42} \mathrm{erg} \mathrm{s}^{-1}$ ); (5) PA of the ionization axis (in deg); (6) ENLR angular extent (in arcsec); (7) ENLR radius (in kpc); (8) $\mathrm{H} \alpha+[\mathrm{N} \mathrm{II}]$ total luminosity, integrated above $3 \sigma_{\text {sky }}$ (in units of $10^{42} \mathrm{erg} \mathrm{s}^{-1}$ ); (9) total ionized gas mass (in units of $10^{8} M_{\odot}$ ); (10) standard deviation of the sky value in the [O III] image in units of $10^{-18} \mathrm{erg} \mathrm{cm}^{-2} \mathrm{~s}^{-1}$.

$\approx 1 \mathrm{kpc}$ west of the nucleus-to the right in Figure 12-in a region where the [O III] flux is well above $3 \sigma_{\text {sky }}$. One possibility is the presence of a region of recent star formation. A similar effect-the presence of regions of recent star formation, although smaller-could explain small decreases in the $[\mathrm{O} \mathrm{III]} / \mathrm{H} \beta$ ratio along the ENLR in other objects. Alternatively, as we have estimated $\mathrm{H} \beta$ from $\mathrm{H} \alpha$, this decrease may be due to reddening decreasing the [O III] flux but less so than the $\mathrm{H} \alpha$ flux, leading to an overestimation of $\mathrm{H} \beta$ relative to $[\mathrm{O} \mathrm{III}]$.

\subsubsection{The Case for Matter-bounded ENLR}

The $[\mathrm{O} \mathrm{III}] / \mathrm{H} \beta$ ratios decrease abruptly beyond the limits of the ENLR, where the [O III] fluxes become lower than $3 \sigma_{\text {sky }}$, while the $[\mathrm{O} \mathrm{III}] / \mathrm{H} \beta$ ratios are remarkably constant throughout the nebulae where the [O III] is above $3 \sigma_{\text {sky }}$, as illustrated by the blue points in Figure 12, suggesting a constant ionization parameter $U$. This can be understood if $Q$ is constant and $n_{e}$ decreases with the distance from the nucleus as $R^{-2}$. Resolved measurements of the gas density along the ionization cone in the nearby Sy 2 galaxy NGC 3281 (Storchi-Bergmann et al. 1992) show exactly this behavior for the gas density, supporting an approximately constant value of $Q$ up to the limits of the nebulae, that we can define as corresponding to where the [O III] flux decreases below $3 \sigma_{\text {sky }}$. This result suggests that the ENLRs in all QSO2s of this study are matter-bounded.

The abrupt decrease in the $[\mathrm{O} \mathrm{III]} / \mathrm{H} \beta$ line ratios at the borders of the ENLR was also pointed out by Liu et al. (2013) in their GMOS-IFU data, arguing that this may imply that the ENLR is matter-bounded by the lack of gas at larger distances, including being bounded by the extent of the disk of the galaxy. This limit- the galaxy radius-should apply if there are no external sources of gas, but it could increase in the cases of availability of gas at larger distances, as seems to be the case in our sample.

Further evidence that the ENLRs of the QSOs of the present study are matter-bounded are present in at least three targets of our sample. (1) In QSO 1, the ionizing radiation escapes the host galaxy and reaches a cloud to the southeast (Figures 3 and 12), ionizing the gas there. This implies that the apparent decrease in the gas excitation between the galaxy and the cloud is not due to the lack of photons but rather to the lack of matter. (2) In QSO 2, the nuclear radiation ionizes gas up to $\sim 10 \mathrm{kpc}$ to the west (right in Figures 4 and 12) and only $\sim 3 \mathrm{kpc}$ to the east (left in the figures). This can be understood as the result of a merger process during which gas is spread between the galaxies and not as much to the other (east) side of the QSO, as seen in Figure 4. Thus, what is limiting the size of ENLRs to the east in Figure 12 is not the rate of ionizing photons but rather the availability of gas in that direction. (3) In QSO 4, the AGN radiation is ionizing a detached cloud to the right in Figure 12 (that could be the remnant of a merger or the result of a previous outflow from the AGN); thus, the decrease in excitation between the QSO and the cloud is due to the lack of gas, indicating that, also in the opposite direction, ionizing photons escape as the nebula is matter-bounded.

The cases above indicate that the ionizing photons of the AGN can reach distances far beyond the apparent limits of the host galaxy as seen in the continuum images; this is also supported by previous studies in the literature, such as the case of the quasar MR 2251-178 (Kreimeyer \& Veilleux 2013) discussed above.

\subsection{The Incidence of Galaxy Interactions}

The continuum images of the QSO hosts of our sample show signatures of interactions in six out of our nine QSOs. In target 1 (Figure 3), there is a structure with an angular size of approximately $1^{\prime \prime} \times 1^{\prime \prime}$ in the continuum image at about $15 \mathrm{kpc}$ to the southeast of the AGN, but, due to the large contamination from gas emission in the continuum image of this galaxy (see Section 4.2.1), we favor that this structure is a gas cloud only appearing in the continuum image because of the [O III] emission-line contribution. In target 2 (Figure 4), the QSO host is in clear interaction with a big companion closer than $\approx 10 \mathrm{kpc}$ to the southwest. In target 3 (Figure 5), the continuum image shows what seems to be a secondary nucleus $\approx 0$ !' 3 from the nucleus to the southeast, suggesting that this may be an advanced stage of a merger. Target 4 (Figure 6) does not show a clear signature of a merger, but there is a cloud to the northwest only seen in gas emission that could be either reminiscent of a merger/interaction or due to a previous outflow from the AGN. In target 6 (Figure 8), the continuum image shows a compact source $\approx 20 \mathrm{kpc}$ to the northwest, with the QSO host showing an asymmetric morphology elongated in this direction that could be a result of an interaction. Target 8 (Figure 10) seems to be in a late stage of a merger with what seems to be remains of a galaxy $\approx 6 \mathrm{kpc}$ to the northwest. 

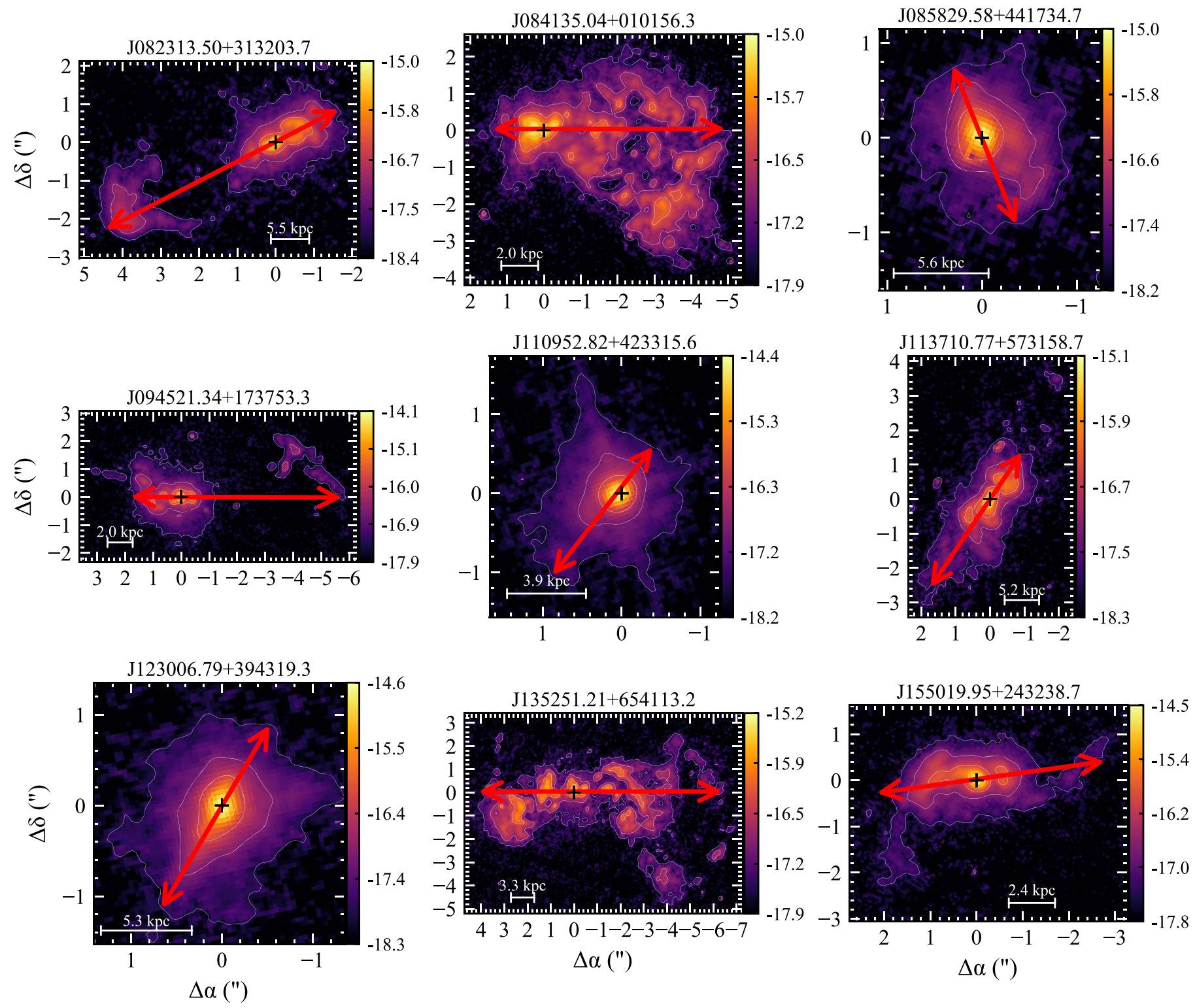

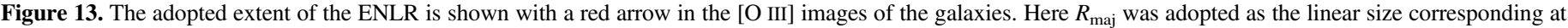

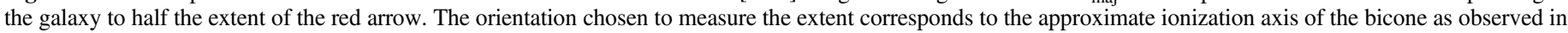
the excitation maps of Figures 3-11 and listed as PA in Table 5. The contour values range from $3 \sigma_{\text {sky }}$ to $F_{\text {max }}$.

Target 9 (Figure 11) also shows what seems to be a small galaxy at $\approx 5 \mathrm{kpc}$ to the south of the nucleus.

In summary, six of the nine QSOs show signatures of mergers or interactions. This frequency of interactions in the sample was not anticipated, as the QSOs were selected only for their proximity -in order to allow resolving of the NLR structure and for their [O III] luminosity-in order to probe a higherluminosity regime than the previous spatially resolved studies. This high incidence of interactions supports the relation between strong nuclear activity (above $\mathrm{L}\left[\mathrm{O}\right.$ III] $=10^{42.5} \mathrm{erg} \mathrm{s}^{-1}$ ) and galaxy mergers, as suggested by previous studies (e.g., Treister et al. 2012; Glikman et al. 2015).

\subsection{Relation between $R_{\text {maj }}$ and $\mathrm{L}[O$ III]}

The analysis of the [O III] images from the HST NLR snapshot survey of Schmitt et al. (2003a, 2003b) revealed a relation between the NLR (or ENLR) extent $R_{\mathrm{NLR}}$ and the AGN luminosity $L([\mathrm{O} \mathrm{III}])$. For a sample of 60 AGNs with
$L([\mathrm{O}$ III $])<10^{42} \mathrm{erg} \mathrm{s}^{-1}$, they found the relation $R_{\mathrm{NLR}} \propto$ $L[\mathrm{O} \mathrm{III}]^{0.33}$. This was interpreted as due to the fact that the ionization parameter is not constant along the NLR (in which case, the slope should be 0.5) and that most of the [O III] emission comes from a low-density region.

A constant ionization parameter had been suggested by previous observations of the NLR of a sample of QSOs that implied a slope of $\sim 0.5$ (Bennert et al. 2002); this was later argued to be unphysical, as it would lead to predicted NLR sizes larger than the extent of the galaxy (Netzer et al. 2004). One possibility raised to explain the steeper slope obtained for the QSOs is possible contamination by H II regions in the QSO host galaxy. More recent ground-based studies of the extent of the NLR in QSO2s (Greene et al. 2011; Hainline et al. 2013; Liu et al. 2013) have used compilations of NLR sizes obtained from ground-based imaging and long-slit spectroscopy, also investigating the slope of the relation between the NLR radius and its luminosity and finding a value $\alpha=0.22$. This has been 


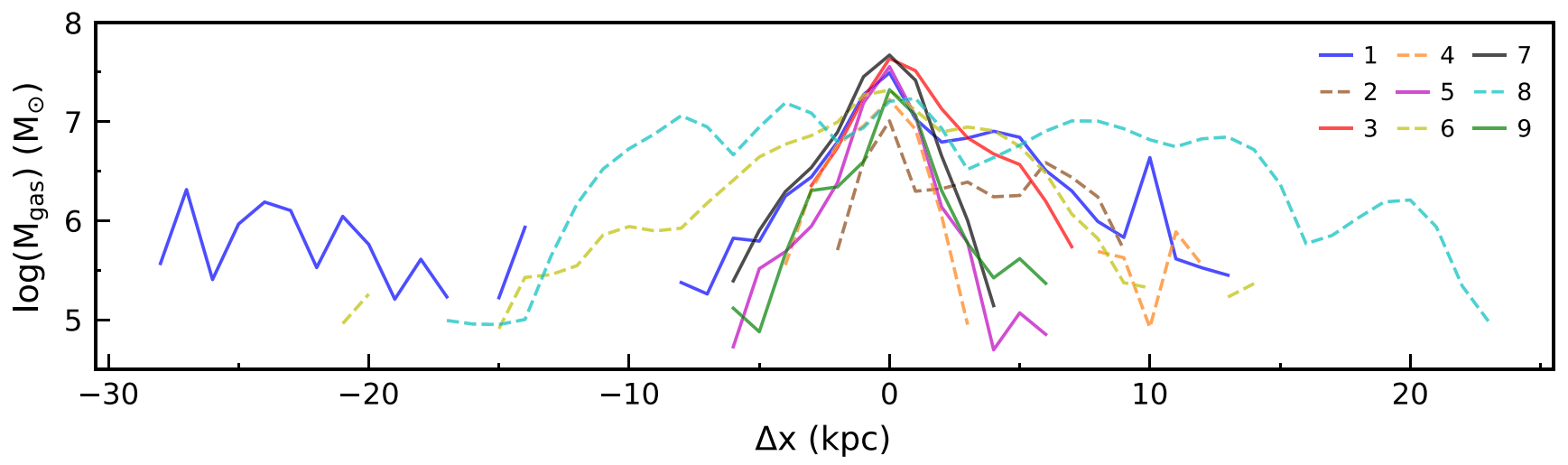

Figure 14. Cumulative gas mass profiles obtained by binning the masses into $1 \mathrm{kpc}^{2}$ at the galaxies, rotating the surface mass density distributions so that the ionization axis is oriented along the $x$-axis, and summing the mass contributions along the perpendicular direction within each kpc.

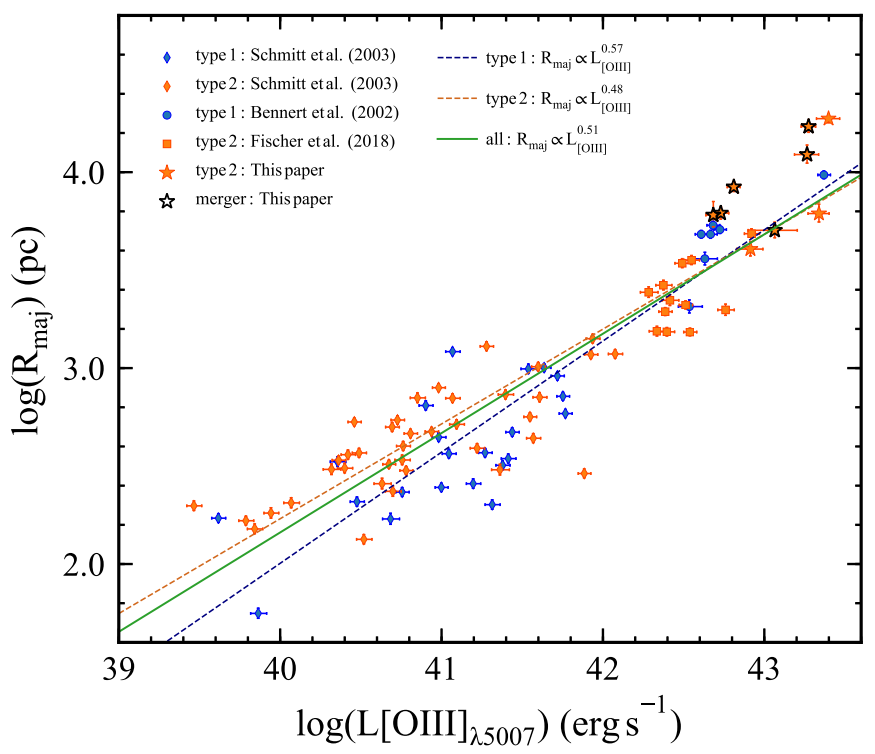

Figure 15. Relation between the extent of the ENLR $R_{\text {maj }}$ and $\log (L[\mathrm{O}$ III] $)$, using data of three samples besides ours (stars)—Schmitt et al. (2003a; diamonds), Bennert et al. (2002; circles), and Fischer et al. (2018; squares) distinguishing type 1 (blue) and type 2 (orange) AGNs, together with the corresponding fits (see the text). The AGNs in our sample that appear to be in interaction are highlighted with black contours.

interpreted as indicating that the NLR size is limited by the density and ionization state of the NLR gas rather than the availability of ionizing photons.

With our measurements, we can now revisit the relation between the ENLR extent and the [O III] luminosity. Combining our measurements of $R_{\text {maj }}$ with those of lower-redshift and luminosity targets from Bennert et al. (2002), Schmitt et al. (2003a), and Fischer et al. (2018), we now span a much larger luminosity range $-39 \leqslant \log (L[\mathrm{O}$ III $]) \leqslant 43.3, L[\mathrm{O}$ III $]$ in $\mathrm{erg} \mathrm{s}^{-1}$ - from which we can derive a new relation. Although this relation has been recently investigated and expanded to higher luminosities by other authors (Greene et al. 2011; Hainline et al. 2013; Liu et al. 2013), as discussed above, these studies used ground-based heterogeneous data that include long-slit spectroscopy and images obtained by different authors with different instruments. At the largest luminosities (and distances) of QSOs, the derived diameters in ground-based studies may be of the order of those of the point-spread functions (PSFs) and thus carry large uncertainties.
The higher-luminosity data we have put together to derive a new relation were all obtained with $H S T$ and thus with at least 10 times better angular resolution than those from groundbased studies. This new relation is shown in Figure 15, where the colors separate AGN type 1 (blue) and type 2 (orange), and our sample is represented by stars. The QSOs in our sample that appear to be in interaction are highlighted with black contours.

Figure 15 shows that $R_{\text {maj }}$ continues to grow with $L[\mathrm{O}$ III] until the maximum luminosity of our sample: $\mathrm{L}[\mathrm{O} \mathrm{III}]=10^{43.3} \mathrm{erg} \mathrm{s}^{-1}$. Linear least-squares regressions were applied to the data using the function curve fit-available in the Scipy library-which also returns a covariance matrix whose diagonal value gives the standard deviation of the parameters fitted. We have obtained three regressions in order to also verify whether we could detect any variation according to the AGN type. The first was restricted to type 1 AGNs, returning the relation

$$
\begin{aligned}
\log \left(R_{\text {maj }}\right)= & (0.57 \pm 0.05) \log L[\mathrm{O} \mathrm{IIII}]_{\lambda 5007} \\
& -20.7 \pm 2.0
\end{aligned}
$$

shown as a blue line in Figure 15. The second was restricted to type 2 AGNs,

$$
\begin{aligned}
\log \left(R_{\text {maj }}\right)= & (0.48 \pm 0.03) \log L[\mathrm{O} \mathrm{III}]_{\lambda 5007} \\
& -17.1 \pm 1.1,
\end{aligned}
$$

shown as an orange line in Figure 15. Finally, using all AGNs, we obtain

$$
\begin{aligned}
\log \left(R_{\text {maj }}\right)= & (0.51 \pm 0.03) \log L[\mathrm{O} \mathrm{IIII}]_{\lambda 5007} \\
& -18.12 \pm 0.98
\end{aligned}
$$

shown as a green line in Figure 15.

These regressions indicate that the dependency of the extent of the ENLR on $L[\mathrm{O} \mathrm{III}]$ is somewhat less steep in type 2 than in type 1 AGNs, although, as there are only a few type 1 AGNs with luminosities $L[\mathrm{O} \mathrm{III}]]>10^{42} \mathrm{erg} \mathrm{s}^{-1}$, this result is not very robust for high luminosities. The last fit, which uses all the data, returns a power of $0.51 \pm 0.03$, which is in agreement with the other two fits within the uncertainties, suggesting that there is no significant difference of this relation for the two types of AGNs.

We have also investigated the effect in the relation of changing the measurements to exclude "detached" gas clouds that may be due to interactions. We considered, in particular, the cases of targets $1,4,8$, and 9 , for which, excluding the most external parts, $R_{\text {maj }}$ values change, respectively, from 18.8 to 
$9.2 \mathrm{kpc}$, from 8.4 to $3.4 \mathrm{kpc}$, from 17.1 to $14.0 \mathrm{kpc}$, and from 6.0 to $3.9 \mathrm{kpc}$. A new fit considering these new values results in

$$
\begin{aligned}
\log \left(R_{\text {maj }}\right)= & (0.49 \pm 0.03) \log L[\mathrm{O} \mathrm{III}]_{\lambda 5007} \\
& -17.4 \pm 0.94,
\end{aligned}
$$

thus consistent with the previous fit within the uncertainties. We prefer to keep the previous fit because detailed imaging, as we have in the present study, is not always available, and in most cases of similar studies, it will not be obvious if the extended gas emission is due to interactions or not.

The relation we have obtained for the whole sample is approximately the $R_{\text {maj }} \propto L[\mathrm{O} \mathrm{IIII}]_{\lambda 5007}^{1 / 2}$ previously obtained by Bennert et al. (2002) for type 1 quasars at similar distances to those of our sources $(0.1 \leqslant z \leqslant 0.4)$, supporting that the ENLR extent continues to grow with the AGN luminosity up to the highest AGN luminosity of our sample of $L[\mathrm{O} \mathrm{IIII}]=10^{43.3} \mathrm{erg} \mathrm{s}^{-1}$. This also seems to imply that the ionization parameter $U$ may be constant, suggesting that the gas density $n_{e}$ decreases with distance from the nucleus $(R)$ as $\sim R^{-2}$. This radial dependence for the gas density has been previously observed in NGC 3281 (StorchiBergmann et al. 1992), as already pointed out, and is close to the $\sim R^{-1.7}$ dependence also found for the NLR of NGC 4151 (Kraemer et al. 2000) but distinct for that observed in other cases in which the kinematics is clearly disturbed due to outflows (e.g., Revalski et al. 2018). It may apply, though, to most of the ENLR, as the outflows seem to be restricted to the inner kpc or so (Fischer et al. 2018).

As pointed out above, Netzer et al. (2004) argued for a limit in the size of the gaseous region ionized by the AGN corresponding to the extent of the galaxy, which makes sense if there is no gas beyond this limit. However, if there is gas spread beyond the galaxy as a consequence of outflows, interactions, or mergers, the extent of the ENLR could still increase with the luminosity, provided that there are enough ionizing photons reaching these outer regions, which seems to be the case in our sample. Our results show that, as the AGN luminosity increases, the extent of the ionized region continues to increase if there is gas to be ionized.

Some ENLRs extending even beyond those studied here have been reported in the literature, as is the case of the quasar MR 2251-178 (Kreimeyer \& Veilleux 2013), discussed in Section 5.1.1, in which the ENLR reaches $\approx 90 \mathrm{kpc}$ from the nucleus. In addition, the authors concluded that the ENLR is still matter-bounded, as they estimated that $65 \%-95 \%$ of the quasar ionizing radiation makes its way out of the system. As pointed out by these authors, this finding highlights the importance that quasar radiative feedback may have on the intergalactic medium and the need for more similar studies.

In a long-slit spectroscopic study of a similar sample to ours, of 12 nearby $(z \sim 0.1)$ QSOs, Sun et al. (2017) reported, for seven galaxies of their sample, NLR extents ranging from 13 to $19 \mathrm{kpc}$, overlapping our largest $R_{\text {maj }}$ values and reaching the same maximum value. But, putting together their data with those from previous works of their group, they argued that the relation between the size of the NLR and the AGN luminosity flattens out at $R_{\mathrm{NLR}} \sim 10 \mathrm{kpc}$. We have not found this flattening in our data.

Alternatively, it may be argued that our sample is somehow "special" due to the high occurrence of interactions that provide gas to be ionized beyond the extent of the galaxies, even though they were selected solely on the basis of distance and luminosity. In addition, it is not obvious, at the corresponding galaxy distances or even larger, how to recognize if the gas origin is previous or ongoing interactions.

\subsection{Total Gas Masses and Surface Mass Densities}

The total masses of ionized gas are very similar among the galaxies, ranging from $0.4 \times 10^{8}$ to $2 \times 10^{8} M_{\odot}$. These masses are 2-3 orders of magnitude larger than those we have been obtaining in integral field spectroscopic studies of nearby $\mathrm{Sy}$ galaxies (about 2 orders of magnitude less luminous) in the optical and near-infrared, although in these nearby cases, the field of view corresponds to smaller regions at the galaxies of just a few kpc (e.g., Riffel et al. 2015, 2018).

On the other hand, the ionized gas masses of our sample are similar to those obtained by Harrison et al. (2014) for a sample of 16 type 2 AGNs of similar luminosities and redshifts to those of our targets and by Tadhunter et al. (2014) and Couto et al. (2017) for radio galaxies at similar redshifts, consistent with them having a similar origin, argued to be gas-rich mergers in Tadhunter et al. (2014).

\subsection{Implications for Feedback}

Our narrowband images show extents for the ENLR ranging from 4 to $19 \mathrm{kpc}$. These can be compared to those of Fischer et al. (2018) for a similar sample of active galaxies also selected from Reyes et al. (2008) at somewhat lower redshifts and luminosities. Fischer et al. (2018) also obtained HST [O III] narrowband images, as in our study, but instead of $\mathrm{H} \alpha+[\mathrm{N} \mathrm{II}]$ images, they obtained long-slit STIS spectroscopy in order to investigate the [O III] gas kinematics. The elongated morphologies of the ENLR are similar to those of our targets but with somewhat lower $R_{\text {maj }}$ values, close to about $5 \mathrm{kpc}$.

The long-slit spectroscopy of Fischer et al. (2018), although obtained only along the ionization axis, revealed a disturbed kinematics for the gas, suggesting outflows-e.g., highvelocity dispersion, double or triple components-only within the inner kpc. Beyond this region, the gas kinematics, instead of outflows, revealed in most cases apparent rotation in the galaxy plane, with blueshifts observed to one side and redshifts to the other side of the nucleus. They measured the distance from the nucleus within which they observed the signature of outflows, calling it $R_{\text {out }}$, finding that, on average, $R_{\text {out }} / R_{\text {maj }}=$ 0.22 , where $R_{\text {maj }}$ is their adopted maximum extent of the ionized gas region (we have adopted the same notation as in their study). Typical outflow velocities are in the range $250-500 \mathrm{~km} \mathrm{~s}^{-1}$.

The fact that the outflow is more compact than the ionized gas region in active galaxies is also a common result of previous studies of local AGNs, such as those of the AGN Integral Field Spectroscopy (AGNIFS) group: Schnorr-Müller et al. (2014a), Lena et al. (2015), Riffel et al. (2015), and Couto et al. (2017), and as has also been pointed out by Fischer et al. (2017), and Revalski et al. (2018), Sun et al. (2017). This can be interpreted as due to the fact that most of the extended [O III] emission comes from gas that is not outflowing but is usually rotating in the plane of the host galaxy.

Although we have not yet observed the gas kinematics of our sample, we can use the information we have gathered via the images and SDSS spectroscopy to estimate the mass outflow rate and the range of powers of the presumed outflows in our objects. In order to do this, we adopted the following assumptions. 
(i) Regarding the extent of the outflow, our sample includes AGNs with higher luminosities than those of Fischer et al. (2018), and it may well be that the outflow extends to larger distances than $R_{\text {out }}$. We thus considered two limits for the extents: an upper limit of $R_{\text {maj }}$ (which we called model $a$ ) and a lower limit of $0.2 R_{\text {maj }}$ (which we called model $b$ ).

(ii) Regarding the outflowing gas mass, we have assumed that all gas mass within the considered radius $\left(R_{\text {maj }}\right.$ or $R_{\text {out }}=$ $\left.0.2 R_{\text {maj }}\right)$ is outflowing.

(iii) Regarding the velocity of the outflow, we have used the [O III] $\lambda 5007$ emission-line profile from the SDSS spectra to obtain the velocity $v_{80}=W_{80} / 1.3$, where $W_{80}$ is the width of the line profile at the height that corresponds to $80 \%$ of the line flux. $W_{80}$ probes the wings of the profile, corresponding to the highest velocities, most probably due to the outflows. We have followed Sun et al. (2017) and adopted $v_{80}$ as the velocity of the outflow. This velocity is listed in the last column of Table 4. In addition, we use the approximation that all spaxels have the same velocity, as we do not have spatial information on the kinematics so far.

We have used three methods to calculate the mass outflow rate and also used this rate to calculate the power of the outflow in order to compare it with the QSO bolometric luminosity $L_{\mathrm{Bol}}$. Here $L_{\mathrm{Bol}}$ was calculated using the relation between the reddening-corrected $L\left[\mathrm{O}\right.$ III] and $L_{\mathrm{Bol}}$ of Trump et al. (2015). We have corrected $L[\mathrm{O}$ III] for reddening according to Equation (1) of Lamastra et al. (2009), in which the average reddening value was obtained from the SDSS-III spectra $\mathrm{H} \alpha / \mathrm{H} \beta$ ratio assuming an intrinsic value of 3.0 (Osterbrock \& Ferland 2006). In order to compare the power of the outflows with $L_{\mathrm{Bol}}$, we have also corrected for reddening, in the calculations below, $L(\mathrm{H} \beta)$ (assumed to have the same correction as [O III] due to the proximity in wavelength between the two lines). These values were then used to obtain reddening-corrected values for $\mathrm{H} \alpha$ and $M_{\mathrm{ENLR}}$.

Method 1. The mass outflow rate was estimated from the ratio between the reddening-corrected mass of the ENLR $M$ and the time $t=R / v_{80}$ it took the gas at the border of the ENLR to reach the maximum distance $R$ from the nucleus $\left(R_{\text {maj }}\right.$ for model $a$ and $0.2 R_{\text {maj }}$ for model $b$ ).

The power of the outflow $d E / d t$ was calculated as

$$
\frac{d E}{d t}=0.5 \frac{M v^{2}}{t}
$$

where the outflow velocity is assumed constant at $v=v_{80}$ and $M$ is the value of $M_{\mathrm{ENLR}}$ from Table 5 corrected for reddening.

Method 2. We use the same equation as above to calculate the power of the outflow, but instead of calculating the mass outflow rate as $M / t$, we use $(d M) /(d t)$ calculated as

$$
\frac{d M}{d t}=m_{p} n_{e} v f A
$$

where $n_{e}$ is the electronic density, assumed to be $100 \mathrm{~cm}^{-3}$ (as a typical value for extended emission-line regions, but see discussion below for the effect of a possibly higher density); $m_{p}$ is the mass of the proton; $f$ is the filling factor; $v$ is the gas velocity, adopted as above $\left(v_{80}\right)$; and $A$ is the cross-section of the outflow.

We have then used the $\mathrm{H} \alpha$ luminosity (obtained as $\eta L([\mathrm{~N}$ II] $+\mathrm{H} \alpha$ ) integrated within the radius considered, $R_{\text {maj }}$ or $R_{\text {out }}$ (for models $a$ and $b$, respectively), and corrected for reddening to derive $f$ :

$$
L(\mathrm{H} \alpha)=j_{\mathrm{H} \alpha} 4 \pi f V,
$$

where $j_{\mathrm{H} \alpha}$ is the $\mathrm{H} \alpha$ emissivity as defined in Osterbrock \& Ferland (2006), and $V$ is the volume of the emitting region. We have first adopted as the geometry of the outflow a cone with base area $A$ and height $R$, assumed to be $R_{\text {maj }}$ in model $a$ and $R_{\text {out }}$ in model $b$. But we have nevertheless concluded that the geometry of the outflow cancels out when you calculate the filling factor; thus, an outflow through a spherical surface with radius $R$ would result in the same expression, as follows:

$$
\dot{M}=\frac{3 m_{p} v L(\mathrm{H} \alpha)}{\left(\frac{j_{\mathrm{H} \alpha}}{j_{\mathrm{H} \beta}}\right) n_{e} \alpha_{\mathrm{H} \beta}^{\mathrm{eff}} h \nu_{\mathrm{H} \beta} R},
$$

with the emissivities $j$ and recombination coefficient $\alpha_{\mathrm{H} \beta}^{\text {eff }}$ values obtained from Osterbrock \& Ferland (2006) for $n_{e}=100 \mathrm{~cm}^{-3}$ and $T=10,000 \mathrm{~K}$. We note that, in this method, the mass outflow rate results are inversely proportional to the gas density. Thus, if the gas density is, for example, five times larger $\left(500 \mathrm{~cm}^{-3}\right)$, the mass outflow rate will be five times smaller. But there is no assumption regarding the filling factor, which is calculated from the data.

Method 3. We have also estimated what can be considered a "maximum" mass outflow rate, using Equation (4) of Sun et al. (2017), that assumes a density, filling factor, and spherical geometry for the flow,

$$
\frac{d M}{d t}=m_{p} n_{e} v 4 \pi R^{2},
$$

where Sun et al. (2017) adopted a constant $n_{e}=0.5 \mathrm{~cm}^{-3}$, which is equivalent, for example, to the product of a density of $n_{e}=100 \mathrm{~cm}^{-3}$ times a filling factor of 0.005 (a typical value, similar to what we have obtained from previous studies) or to a somewhat larger density of $n_{e}=500 \mathrm{~cm}^{-3}$ times a smaller filling factor of 0.001 .

The power of the outflow is again obtained using Equation (8), replacing $M / t$ with $d M / d t$ calculated as above.

The resulting mass outflow rates and powers are shown in Table 6 for the three methods above for each QSO. In the first line, we show the power calculated assuming that the extent of the outflow is $R_{\text {maj }}$, and in the second line, we assume that it is $R_{\text {out }}$. We have also included in the table an estimate of the ratio between the outflow powers and the bolometric luminosity of the AGN, $\dot{E} / L_{\mathrm{bol}}$, where $L_{\mathrm{bol}}$ was calculated from the $L[\mathrm{O}$ III] value corrected for reddening using the bolometric correction given by Equation (9) of Trump et al. (2015).

The values of the mass outflow rates $\dot{M}$ range from $\sim 5$ to $\sim 200 M_{\odot} \mathrm{yr}^{-1}$ for the first method and from $\sim 15$ to $\sim 600 \mathrm{M}_{\odot} \mathrm{yr}^{-1}$ for the second method. A higher density of $500 \mathrm{~cm}^{-3}$ will result in a mass outflow rate five times lower, thus from $\sim 1$ to $\sim 40 M_{\odot} \mathrm{yr}^{-1}$ for the first method and $\sim 3$ to $\sim 100 M_{\odot} \mathrm{yr}^{-1}$ for the second method. These values range from being of the same order up to two orders of magnitude larger than those of our previous studies of closer and less luminous AGNs (e.g., Storchi-Bergmann et al. 2010; Riffel et al. 2013; Barbosa et al. 2014). In the work of Revalski et al. (2018), in which the authors calculated the mass outflow rate as a function of distance from the nucleus in the NLR of the Sy 2 galaxy 


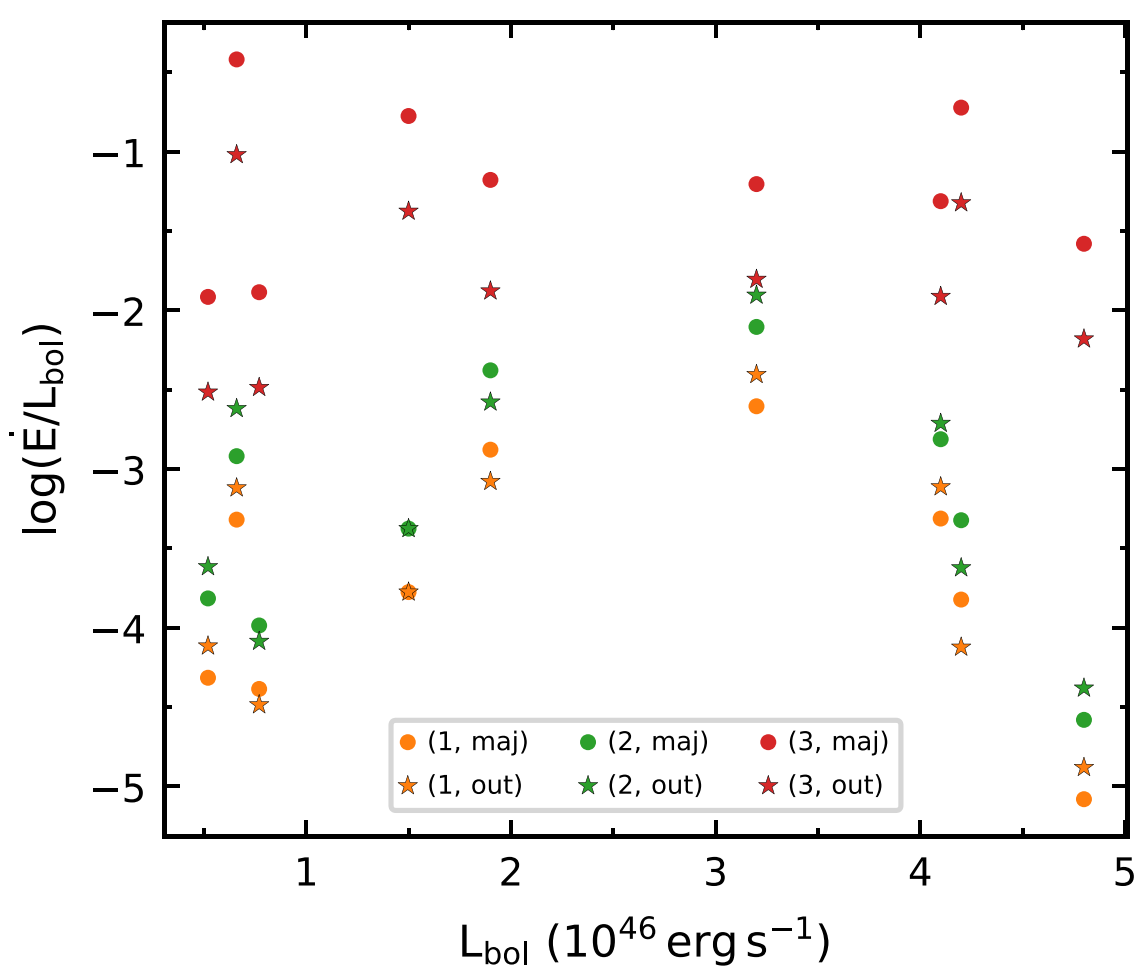

Figure 16. Ratio between the outflow powers $\dot{E}$ and the AGN bolometric luminosity $L_{\mathrm{bol}}$ as a function of $L_{\mathrm{bol}}$ for methods 1 (yellow), 2 (green), and 3 (red) used to calculate $\dot{E}$ (see the text). The circles correspond to an extent of the outflow of $R_{\text {maj, }}$, while the stars correspond to an extent of $R_{\text {out }}=0.2 \times R_{\text {maj }}$ (see the text).

Table 6

Mass Outflow Rates and Outflow Power According to Three Different Methods

\begin{tabular}{|c|c|c|c|c|c|c|c|c|c|c|}
\hline \multirow[b]{2}{*}{ No. } & \multirow[b]{2}{*}{$R$} & \multicolumn{3}{|c|}{ Method 1} & \multicolumn{3}{|c|}{ Method 2} & \multicolumn{3}{|c|}{ Method 3} \\
\hline & & $\log (\dot{M})$ & $\log (\dot{E})$ & $\log \left(\dot{E} / L_{\mathrm{bol}}\right)$ & $\log (\dot{M})$ & $\log (\dot{E})$ & $\log \left(\dot{E} / L_{\mathrm{bol}}\right)$ & $\log (\dot{M})$ & $\log (\dot{E})$ & $\log \left(\dot{E} / L_{\mathrm{bol}}\right)$ \\
\hline 1 & $R_{\text {maj }}$ & 0.9 & 41.6 & -5.1 & 1.4 & 42.1 & -4.6 & 4.4 & 45.1 & -1.6 \\
\hline \multirow[t]{2}{*}{2} & $R_{\text {maj }}$ & 0.8 & 41.5 & -4.4 & 1.3 & 41.9 & -3.9 & 3.3 & 44.0 & -1.9 \\
\hline & $R_{\text {out }}$ & 0.7 & 41.4 & -4.5 & 1.2 & 41.8 & -4.1 & 2.7 & 43.4 & -2.5 \\
\hline \multirow[t]{2}{*}{3} & $R_{\text {maj }}$ & 1.9 & 43.4 & -2.9 & 2.4 & 43.9 & -2.4 & 3.6 & 45.1 & -1.2 \\
\hline & $R_{\text {out }}$ & 1.7 & 43.2 & -3.1 & 2.2 & 43.7 & -2.6 & 3.0 & 44.4 & -1.8 \\
\hline \multirow[t]{2}{*}{5} & $R_{\text {maj }}$ & 2.2 & 43.9 & -2.6 & 2.6 & 44.4 & -2.1 & 3.5 & 45.3 & -1.2 \\
\hline & $R_{\text {out }}$ & 2.3 & 44.1 & -2.4 & 2.8 & 44.6 & -1.9 & 2.9 & 44.7 & -1.8 \\
\hline \multirow[t]{2}{*}{6} & $R_{\text {maj }}$ & 1.1 & 42.4 & -3.8 & 1.6 & 42.8 & -3.3 & 4.2 & 45.4 & -0.7 \\
\hline & $R_{\text {out }}$ & 1.1 & 42.4 & -3.8 & 1.6 & 42.8 & -3.3 & 3.6 & 44.8 & -1.3 \\
\hline \multirow[t]{2}{*}{7} & $R_{\text {maj }}$ & 1.8 & 43.3 & -3.3 & 2.3 & 43.8 & -2.8 & 3.8 & 45.3 & -1.3 \\
\hline & $R_{\text {out }}$ & 2.0 & 43.5 & -3.2 & 2.4 & 43.9 & -2.7 & 3.2 & 44.7 & -1.9 \\
\hline
\end{tabular}

Note. Here $R$ is the adopted extent of the outflow, $\dot{M}$ is the mass outflow rate in units of $M_{\odot} \mathrm{yr}^{-1}$, and the power of the outflow $\dot{E}$ is in units of erg s ${ }^{-1}$.

Mrk 573, the highest value of $3.4 \pm 0.5 M_{\odot} \mathrm{yr}^{-1}$ is obtained at $210 \mathrm{pc}$ from the nucleus.

The corresponding estimated powers of the outflows (for methods 1 and 2) are in the range $10^{41.5}<\log (\dot{E})<10^{44.5}$ (in erg s $\left.{ }^{-1}\right)$ and correspond to a range of $-5<\log \left(\dot{E} / L_{\mathrm{Bol}}\right)<$ -2 , with only three cases in which it is larger than the $0.5 \%$ threshold corresponding to a significant impact on the host galaxy (e.g., Hopkins \& Elvis 2010). These $\dot{E} / L_{\mathrm{Bol}}$ values are plotted as a function of $L_{\mathrm{Bol}}$ in Figure 16 in yellow and green symbols for methods 1 and 2 , respectively. They increase between $L_{\mathrm{Bol}}=0.5 \times 10^{46}$ and $3.5 \times 10^{46} \mathrm{erg} \mathrm{s}^{-1}$ but then decrease for higher luminosities.

It is important to point out that our calculations refer only to ionized gas emitting in the optical spectral window and do not consider the possible presence of neutral and molecular gas. Recent studies (e.g., Fiore et al. 2017) suggest that molecular gas is the dominant gas phase surrounding nearby AGN nuclei, with masses that can be $\sim 2$ orders of magnitude larger than that 
of ionized gas. If such molecular gas is present in our sample and also outflowing, the corresponding power of the outflow will be much larger than than the above and will most probably exceed the $0.5 \%$ threshold.

When using Method 3, in which the gas mass is not constrained by $L(\mathrm{H} \alpha)$ and the geometry is assumed to be spherical, the mass outflow rates reach values 2-3 orders of magnitude higher than those in Methods 1 and 2, corresponding to thousands of $M_{\odot} \mathrm{yr}^{-1}$. These mass outflow rates lead to estimated outflow powers that are above the $0.5 \%$ threshold for most QSOs. The $\dot{E} / L_{\mathrm{Bol}}$ values for Method 3 are plotted as a function of $L_{\mathrm{Bol}}$ in Figure 16 in red symbols. There seems to be no relation between these two quantities. As the mass outflow rate in this case is proportional to $R^{2}$, the resulting power is also proportional to $R^{2}$, and thus those calculated using $R_{\text {maj }}$ as the extent of the outflows are five times higher than those calculated using $R_{\text {out }}$, while this does not happen in the cases of Methods 1 and 2.

\section{Conclusions}

We have presented HST continuum, [O III] $\lambda 5007$, and $\mathrm{H} \alpha$ $+[\mathrm{N} \mathrm{II}]$ narrowband images, as well as $[\mathrm{O} \mathrm{III}] / \mathrm{H} \beta$ excitation maps, and obtained the extent, morphology, and masses of the ionized gas in the ENLR of nine type 2 AGNs classified as QSO2 due to their high luminosities ( $\log \mathrm{L}[\mathrm{O}$ III] $>42.5, L$ in erg $\mathrm{s}^{-1}$ ). Under the assumption that the ionized gas is outflowing, we have also estimated the corresponding mass outflow rates and powers. Our main findings are as follows.

1. The ENLR is spatially resolved in all galaxies, and its extent ranges from 4 to $19 \mathrm{kpc}$; the largest extents may be related to the fact that six of the nine galaxies seem to be in interaction, with gas emission extending well beyond the body of the galaxy seen in the continuum maps.

2. The ENLR morphology is clearly elongated and bipolar in six of the nine galaxies, and excitation maps [O III]/ $(\mathrm{H} \alpha+[\mathrm{N} \mathrm{II}])$ show a biconical structure, with the highest excitation gas along the ionization axis and decreasing with increasing angular distance from it. The exceptional HST angular resolution was key to revealing this structure.

3. The high emission-line ratios obtained along the ionization axes $([\mathrm{O} \mathrm{III}] / \mathrm{H} \beta \geqslant 10)$ and the low values obtained perpendicular to it through the nucleus $([\mathrm{O} \mathrm{III}] / \mathrm{H} \beta \leqslant 7$ ) imply that the rate of ionizing photons escaping along the ionization axis is $\approx 10$ times higher than that escaping perpendicular to it.

4. The above result supports the presence of an obscuring torus that can thus survive the corresponding AGN luminosities up to $\log (L[\mathrm{O} \mathrm{III}])=43.3\left(L_{\text {in }} \operatorname{erg~s}^{-1}\right)$.

5 . We have revisited the relation between the extent of the $\operatorname{ENLR}\left(R_{\text {maj }}\right)$ and $L[\mathrm{O} \mathrm{III}]$, finding that $R_{\text {maj }}$ increases with $L[\mathrm{O}$ III] over the range $39.5<\log [\mathrm{O}$ III] $<43.5(L$ in $\left.\mathrm{erg} \mathrm{s}^{-1}\right)$ as $\log \left(R_{\text {maj }}\right)=(0.51 \pm 0.03) \log (L[\mathrm{O}$ III $])-$ $18.12 \pm 0.98$.

6. We do not find a flattening in the above relation up to $L$ $[\mathrm{O}$ III $]=43.3\left(L\right.$ in $\left.\operatorname{erg~s}^{-1}\right)$, implying that the ENLR in luminous AGNs can extend to distances beyond the limit of the galaxy if there is availability of gas, e.g., from outflows or interactions, as seems to be the case for most galaxies of our sample.
7. We attribute the flattening of the above relation reported in previous studies to the fact that the ENLR is matterbounded in most AGN hosts. The constancy of the $[\mathrm{O} \mathrm{III]} / \mathrm{H} \beta$ along the ENLR observed in our targets supports that it is matter-bounded even in them, implying that the AGN radiation is still escaping to the intergalactic medium and does so in most luminous AGNs.

8. The ionized gas masses of the ENLR range from 0.4 to $2 \times 10^{8} M_{\odot}$; much of it may have been acquired in an interaction and thus seems to be more related to the feeding of the AGN than to its feedback, but this can only be concluded via resolved spectroscopy of the ENLR to probe its resolved kinematics. This will be our next step following the present study.

9. Although we are still in the process of acquiring resolved spectroscopy of our targets, we have estimated the NLR outflow velocities from the widths $W_{80}$ of the [O III] emission-line profiles in SDSS spectra, combined with the above ENLR masses and extents to estimate the mass outflow rates and power of the outflows using three different methods, as follows.

10. For the first two methods, in which the ionized gas masses are constrained by the measured $\mathrm{H} \alpha$ luminosity, we obtain mass outflow rates in ionized gas in the range $\sim 1-100 M_{\odot} \mathrm{yr}^{-1}$.

11. The corresponding powers of the outflows are larger than $0.5 \% L_{\mathrm{Bol}}$ - the usually adopted threshold for a significant impact on the host galaxy-for only four QSOs of the sample; on the other hand, we point out that our calculations do not take into account other gas phases, like neutral and molecular gas (that seems to be the dominant phase in many nearby AGNs), that will lead to larger feedback powers, if present.

12. Using a third method in which the gas masses are not constrained by the $\mathrm{H} \alpha$ luminosity and the assumed outflow geometry is spherical, we obtain much larger mass outflow rates that reach thousands of $M_{\odot} \mathrm{yr}^{-1}$, with corresponding outflow powers exceeding $0.5 \% L_{\mathrm{Bol}}$ for most QSOs.

We plan to revisit the above calculations in a forthcoming paper using resolved spectroscopy of our targets, from which we will obtain gas densities and temperatures and model the ionization structure of the ENLR. We are now in the process of acquiring such data.

We thank the referee for the numerous valuable suggestions that led to a much improved discussion of our results. This work has used data from the Hubble Space Telescope, which is operated by AURA for NASA, as well as from the Sloan Digital Sky Survey. Funding for SDSS-III has been provided by the Alfred P. Sloan Foundation, the Participating Institutions, the National Science Foundation, and the U.S. Department of Energy Office of Science. The SDSS-III website is http://www.sdss3.org/. TSB acknowledges support from the Brazilian funding agencies CNPq, CAPES, and FAPERGS. The submission of the HST proposal and the corresponding data were partially acquired in a visit by TSB to the Harvard/ Smithsonian Center for Astrophysics. LFLM was financed in part by Coordenação de Aperfeiçoamento de Pessoal de Nível Superior-Brasil (CAPES; grant 88888.040697/2013-00). 


\section{ORCID iDs}

T. Storchi-Bergmann (1) https://orcid.org/0000-0003-1772-0023

H. R. Schmitt (1) https://orcid.org/0000-0001-7376-8481

T. C. Fischer (10 https://orcid.org/0000-0002-3365-8875

S. Kraemer (1) https://orcid.org/0000-0002-6928-9848

M. Crenshaw (i) https://orcid.org/0000-0002-6465-3639

P. Maksym iㅏ https://orcid.org/0000-0002-2203-7889

M. Elvis (ib https://orcid.org/0000-0001-5060-1398

G. Fabbiano (1) https://orcid.org/0000-0002-3554-3318

\section{References}

Antonucci, R. 1993, ARA\&A, 31, 473

Baldwin, J. A., Phillips, M. M., \& Terlevich, R. 1981, PASP, 93, 5

Barbosa, F. K. B., Storchi-Bergmann, T., McGregor, P., Vale, T. B., \& Rogemar Riffel, A. 2014, MNRAS, 445, 2353

Bennert, N., Falcke, H., Schulz, H., Wilson, A. S., \& Wills, B. J. 2002, ApJL, 574, L105

Bland-Hawthorn, J., Maloney, P. R., Sutherland, R. S., \& Madsen, G. J. 2013, ApJ, 778, 58

Capetti, A., Axon, D. J., Macchetto, F., Sparks, W. B., \& Boksenberg, A. 1996, ApJ, 469, 554

Ciotti, L., Ostriker, J. P., \& Proga, D. 2010, ApJ, 717, 708

Couto, G. S., Storchi-Bergmann, T., \& Schnorr-Müller, A. 2017, MNRAS, 469, 1573

Crenshaw, D. M., Kraemer, S. B., Gabel, J. R., et al. 2003, ApJ, 594, 116

Crenshaw, D. M., Kraemer, S. B., Schmitt, H. R., et al. 2010, AJ, 139, 871

Elitzur, M., Ho, L. C., \& Trump, J. R. 2014, MNRAS, 438, 3340

Elvis, M. 2000, ApJ, 545, 63

Fabian, A. C. 2012, ARA\&A, 50, 455

Falcke, H., Wilson, A. S., \& Simpson, C. 1998, ApJ, 502, 199

Ferrarese, L., \& Ford, H. 2005, SSRv, 116, 523

Ferruit, P., Wilson, A. S., \& Mulchaey, J. 2000, ApJS, 128, 139

Fiore, F., Feruglio, C., Shankar, F., et al. 2017, A\&A, 601, A143

Fischer, T. C., Crenshaw, D. M., Kraemer, S. B., \& Schmitt, H. R. 2013, ApJS, 209, 1

Fischer, T. C., Kraemer, S. B., Schmitt, H. R., et al. 2018, ApJ, 856, 102

Fischer, T. C., Machuca, C., Diniz, M. R., et al. 2017, ApJ, 834, 30

Fox, A. J., Bordoloi, R., Savage, B. D., et al. 2015, ApJL, 799, L7

Glikman, E., Simmons, B., Mailly, M., et al. 2015, ApJ, 806, 218

Greene, J. E., Zakamska, N. L., Ho, L. C., \& Barth, A. J. 2011, ApJ, 732, 9

Hainline, K. N., Hickox, R., Greene, J. E., Myers, A. D., \& Zakamska, N. L. 2013, ApJ, 774, 145

Haniff, C. A., Wilson, A. S., \& Ward, M. J. 1988, ApJ, 334, 104
Harrison, C. M., Alexander, D. M., Mullaney, J. R., \& Swinbank, A. M. 2014, MNRAS, 441, 3306

Hickox, R. C., Mullaney, J. R., Alexander, D. M., et al. 2014, ApJ, 782, 9

Hopkins, P. F., \& Elvis, M. 2010, MNRAS, 401, 7

Hopkins, P. F., \& Quataert, E. 2010, MNRAS, 407, 1529

Kormendy, J., \& Ho, L. C. 2013, ARA\&A, 51, 511

Kraemer, S. B., Crenshaw, D. M., Hutchings, J. B., et al. 2000, ApJ, 531, 278

Kreimeyer, K., \& Veilleux, S. 2013, ApJL, 772, L11

Lamastra, A., Bianchi, S., Matt, G., et al. 2009, A\&A, 504, 73

Lena, D., Robinson, A., Storchi-Bergman, T., et al. 2015, ApJ, 806, 84

Liu, G., Zakamska, N. L., Greene, J. E., Nesvadba, N. P. H., \& Liu, X. 2013, MNRAS, 436, 2576

Netzer, H., Shemmer, O., Maiolino, R., et al. 2004, ApJ, 614, 558

Novak, G. S., Ostriker, J. P., \& Ciotti, L. 2011, ApJ, 737, 26

Osterbrock, D. E., \& Ferland, G. J. 2006, Astrophysics of gaseous nebulae and active galactic nuclei (Sausalito, CA: University Science Books)

Peterson, B. M. 1997, An Introduction to Active Galactic Nuclei (Cambridge: Cambridge Univ. Press)

Pogge, R. W. 1988, ApJ, 328, 519

Revalski, M., Crenshaw, D. M., Kraemer, S. B., et al. 2018, ApJ, 856, 46

Reyes, R., Zakamska, N. L., Strauss, M. A., et al. 2008, AJ, 136, 2373

Riffel, R. A., Storchi-Bergmann, T., \& Riffel, R. 2015, MNRAS, 451, 3587

Riffel, R. A., Storchi-Bergmann, T., Riffel, R., et al. 2018, MNRAS, 474, 1373

Riffel, R. A., Storchi-Bergmann, T., Winge, C., et al. 2008, MNRAS, 385, 1129

Riffel, R. A., Storchi-Bergmann, T., \& Winge, C. 2013, MNRAS, 430, 2249

Schmitt, H. R., Donley, J. L., Antonucci, R. R. J., et al. 2003a, ApJ, 597, 768

Schmitt, H. R., Donley, J. L., Antonucci, R. R. J., Hutchings, J. B., \& Kinney, A. L. 2003b, ApJS, 148, 327

Schnorr-Müller, A., Storchi-Bergmann, T., Nagar, N. M., et al. 2014a, MNRAS, 437, 1708

Schnorr-Müller, A., Storchi-Bergmann, T., Nagar, N. M., \& Ferrari, F. 2014b, MNRAS, 438, 3322

Sharp, R. G., \& Bland-Hawthorn, J. 2010, ApJ, 711, 818

Storchi-Bergmann, T., Lopes, R. D. S., McGregor, P. J., et al. 2010, MNRAS, 402,819

Storchi-Bergmann, T., Wilson, A. S., \& Baldwin, J. A. 1992, ApJ, 396, 45

Su, M., Slatyer, T. R., \& Finkbeiner, D. P. 2010, ApJ, 724, 1044

Sun, A.-L., Greene, J. E., \& Zakamska, N. L. 2017, ApJ, 835, 222

Tadhunter, C., Dicken, D., Morganti, R., et al. 2014, MNRAS, 445, L51

Treister, E., Schawinski, K., Urry, C. M., \& Simmons, B. D. 2012, ApJL, 758, L39

Trump, J. R., Sun, M., Zeimann, G. R., et al. 2015, ApJ, 811, 26

Veilleux, S., \& Osterbrock, D. E. 1987, ApJS, 63, 295

Wang, J., Fabbiano, G., Karovska, M., et al. 2009, ApJ, 704, 1195

Wilson, A. S., Braatz, J. A., Heckman, T. M., Krolik, J. H., \& Miley, G. K. 1993, ApJL, 419, L61

Wilson, A. S., \& Tsvetanov, Z. I. 1994, AJ, 107, 1227 